\title{
LMC self-lensing from a new perspective
}

\author{
L. Mancini ${ }^{1,2,4}$, S. Calchi Novati ${ }^{1}$, Ph. Jetzer ${ }^{1}$, and G. Scarpetta ${ }^{2,3,4}$ \\ ${ }^{1}$ Institut für Theoretische Physik der Universität Zürich, 8057 Zürich, Switzerland \\ e-mail: novatic@physik.unizh.ch \\ 2 Dipartimento di Fisica "E. R. Caianiello", Università di Salerno, 84081 Baronissi (SA), Italy \\ 3 International Institute for Advanced Scientific Studies, Vietri sul Mare (SA), Italy \\ ${ }^{4}$ Istituto Nazionale di Fisica Nucleare, sez. Napoli, Italy
}

Received 17 October 2003 / Accepted 6 July 2004

\begin{abstract}
We present a new analysis of the location of the observed microlensing events in the direction of the Large Magellanic Cloud (LMC). This is carried out starting from a recently drawn coherent picture of the geometrical structure and dynamics of the LMC disk and by considering different configurations for the LMC bar. In this framework it clearly emerges that the spatial distribution of the events observed so far shows a near-far asymmetry. This turns out to be compatible with the optical depth calculated for the LMC halo objects. Our main conclusion, supported by a statistical analysis of the outcome of an evaluation of the microlensing rate, is that self-lensing cannot account for all the observed events. Finally we propose a general inequality to calculate quickly an upper limit to the optical depth along a line of view through the LMC center.
\end{abstract}

Key words. gravitational lensing - cosmology: dark matter - galaxies: Magellanic Clouds

\section{Introduction}

The microlensing surveys towards the Large Magellanic Cloud (LMC) (Alcock et al. 2000a; Lasserre et al. 2000) have demonstrated the existence of compact objects that act as gravitational lenses somewhere between us and the LMC. In some cases the distance and the mass of the lenses have been determined, thanks to the proper motion of the lens observed by the Hubble Space Telescope (HST) (Alcock et al. 2001a; Gould 2004; Drake et al. 2004) or to the additional information carried by binary systems (Alcock et al. 2000b, 2001b). However, these are special cases since for most events only the duration and the position on the sky plane have been measured. This information is not enough to establish definitively if the detected events are really caused by white dwarfs or MACHOs in the halo of the Milky Way (MW), or are due to stars or MACHOs within the LMC itself.

The survey of the MACHO team indicates a most probable Galactic halo fraction of $20 \%$, with limits of $5 \%$ to $50 \%$ at the $95 \%$ confidence level, assuming that all the events are due to halo lenses. The preferred value for the lens mass is $\sim 0.4 M_{\odot}$. This is consistent with the EROS survey results, which are given however as an upper limit for the Galactic halo fraction.

An interesting alternative is that of "self-lensing", where both source and lens belong to the luminous part of the LMC as suggested by Sahu (1994) and Wu (1994). However, the initial estimates of the optical depth and microlensing rate were lower than the measured values (Gould 1995;
Alcock et al. 1997, 2000a). The self-lensing explanation has been analyzed further going beyond the hypothesis of a "simple" geometry for the LMC with coplanar disk and bar, so that their relative distance would enhance the optical depth and, therefore, the rate. In the model of Zhao \& Evans (2000) the disk and bar stars are on two distinct planes with different inclinations, so that stars on the front plane could lens those in the plane $\approx 1 \mathrm{kpc}$ behind. Besides the morphology, another aspect considered has been the dynamics of the luminous components within the LMC. Aubourg et al. (1999), by using a model which takes into account the correlation between the mass of the lenses in the LMC and their velocity dispersion, have been able to reproduce a self-lensing optical depth, event rate and event duration distribution compatible with the observed ones. Yet objections to this model have been raised by different authors (Gyuk et al. 2000; Alves \& Nelson 2000), especially with respect to the adopted distribution and velocity dispersion of the lensing stars, which seem to be inconsistent with the observations.

The analysis of Jetzer et al. (2002, hereafter Paper I) has shown that the observed events are probably distributed among different components (disk, spheroid and Galactic halo, the LMC halo and self-lensing). This means that the lenses do not all belong to the same population and their astrophysical features can differ greatly from one to the other.

In this paper we address once more the question of the presence of a self-lensing component within the LMC itself. To this end a correct knowledge of the structure and dynamics of the luminous components (disk and bar) of the LMC is essential. 
Here we take advantage of some recent studies of the LMC disk (see Sect. 2.1), while we allow for different geometries for the still poorly known bar component, to calculate the main microlensing quantities. Moreover, with respect to Paper I, based on the moment method (de Rújula et al. 1991), we perform instead a statistical analysis starting from the differential rate of the microlensing events.

The paper is organized as follows: in Sects. 2 and 3 we discuss the LMC morphology and present the models we use to describe the spatial density of the MW halo and of the various components of the LMC. Section 4 is devoted to the calculation of the microlensing quantities, the optical depth and the microlensing rate, as well as to a statistical analysis of the self-lensing events. In Sect. 5 we discuss the spatial asymmetry with respect to the line of nodes of the observed microlensing events. An improved inequality for the optical depth for selflensing by a stellar disk is derived in Sect. 6. We conclude in Sect. 7 with a summary of our results.

\section{Observational data}

\subsection{LMC disk morphology}

Recently, in a series of three interesting papers (van der Marel \& Cioni 2001; van der Marel 2001; van der Marel et al. 2002), a new coherent picture of the geometrical structure and dynamics of the LMC disk has been given. By using the data from

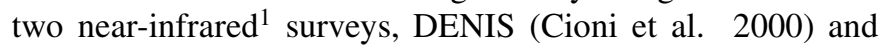
2MASS (Cutri et al. 2000) stellar catalogs, the LMC geometry has been constrained by carefully studying the star count map and the characteristic apparent magnitudes of Asymptotic Giant Branch (AGB) and Red Giant Branch (RGB) stars. The results have been further improved by a detailed and sophisticated study of the LMC line-of-sight velocity field, coupled with a multi-parameter fit of the available velocities of 1041 carbon stars. A basic assumption is that the carbon star population is representative for the bulk of the LMC stars.

The first important conclusion is that the intrinsic shape of the LMC disk is not circular, as assumed before, but elliptical, with an intrinsic ellipticity $\epsilon^{\prime \prime}=0.312 \pm 0.007$. The inclination angle of the LMC disk plane is $i=34.7^{\circ} \pm 6^{\circ}$ and the lineof-nodes position angle is $\Theta=129.9^{\circ} \pm 6^{\circ}$. This value is quite different from the LMC disk major axis position angle, $\mathrm{PA}_{\text {maj }}=$ $189.3^{\circ} \pm 1.4^{\circ}$, corresponding to a position angle $\Phi=202.7^{\circ} \pm$ $1.9^{\circ}$ when measured in the equatorial plane of the LMC disk, starting from the axis pointing towards the North. The radial number density profile along the major axis follows, to lowest order, an exponential profile with an intrinsic scale length equal to $1.54 \mathrm{kpc}$.

A second important conclusion is that the center of mass (CM) of the carbon stars is consistent with the center of the bar and with the center of the outer isophotes of the LMC. As a consequence, the idea of using the distribution of neutral gas as good tracer for the disk stars, which leads to an incorrect LMC model, must be abandoned. The obtained values of the right ascension and declination of the $\mathrm{CM}$, given in $\mathrm{J} 2000$, are

\footnotetext{
${ }^{1}$ Near-infrared surveys provide a very accurate view, thanks to their insensitivity to the dust absorption.
}

$\alpha_{\mathrm{CM}}=5^{\mathrm{h}} 27.6^{\mathrm{m}} \pm 3.9^{\mathrm{m}}, \delta_{\mathrm{CM}}=-69.87^{\circ} \pm 0.41^{\circ}$. The weighted mean of the rotation velocity in the range $4-8.9 \mathrm{kpc}$, where the rotation curve is approximately flat, is $V=49.8 \pm 15.9 \mathrm{~km} \mathrm{~s}^{-1}$, about $40 \%$ lower than the previously inferred and accepted value. Taking into account the asymmetric drift effect, the circular velocity has been corrected and estimated to be equal to $V_{\text {circ }}=64.8 \pm 15.9 \mathrm{~km} \mathrm{~s}^{-1}$. The line-of-sight velocity dispersion has an average value $\sigma=20.2 \pm 0.5 \mathrm{~km} \mathrm{~s}^{-1}$, and is little dependent on the radius. The rate of inclination change is

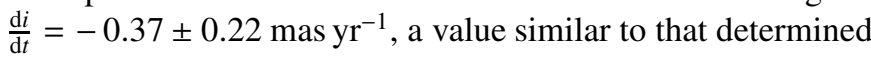
from N-body simulations by Weinberg (2000), which predicts the LMC disk precession and nutation to be due to tidal torques generated by our Galaxy.

A third important conclusion is that the LMC disk has a considerable vertical thickness, in agreement also with the numerical simulations of Weinberg (2000). The thickening of the LMC disk is due to the gravitational interaction with the MW. The ratio $\frac{V}{\sigma}=2.9 \pm 0.9$ is even lower than the corresponding value for the MW thick disk $\left(\frac{V}{\sigma} \simeq 3.9\right)$. The LMC disk is also flared. The best fit of the observed velocity dispersion profile with isothermal disk models, whose vertical density profile is proportional to $\operatorname{sech}^{2}\left(\frac{z}{z_{0}}\right)$, confirms the result found by Alves \& Nelson (2000) that the scale height must increase with radius. The vertical thickening is also in agreement with the results of Olsen \& Salyk (2002), who argued that the LMC contains features that extend up to $2.5 \mathrm{kpc}$ out of the plane.

Let us note that recently some of these conclusions have been challenged by Nikolaev et al. (2004), whose analysis is based on a combination of the results of the MACHO collaboration on the LMC Cepheids with the 2MASS All-Sky Release Catalog.

\subsection{LMC dark halo}

Another important conclusion of the van der Marel et al. analysis is connected with the LMC dark halo. Comparing the LMC total mass enclosed in a sphere of $8.9 \mathrm{kpc}$, dynamically inferred to be $(8.7 \pm 4.3) \times 10^{9} M_{\odot}$, with the visible mass $M_{\mathrm{vis}} \approx 2.7 \times 10^{9} M_{\odot}$, and the mass of neutral gas $\approx 0.5 \times 10^{9} M_{\odot}$ (Kim et al. 1998), van der Marel et al. (2002) have concluded that the LMC must be surrounded by a dark halo with a radius equal to the LMC tidal radius $r_{\mathrm{t}}=15.0 \pm 4.5 \mathrm{kpc}$, a value higher than that of $11 \mathrm{kpc}$, estimated by Weinberg (2000). Moreover, at a distance of $5 \mathrm{kpc}$ from the center of the LMC, the ratio between the tidal force and the LMC self gravitational attraction is reduced by $20 \%$. Thus, one expects that tidal effects influence strongly the structure of the LMC halo, probably causing an elongation of it.

\section{Models}

We adopt the same coordinate systems and notations as in van der Marel et al. (2002). The position of any point in space is uniquely determined by the two angles, right ascension $\alpha$ and declination $\delta$, and by its distance $D$. We introduce also a cartesian coordinate system $(x, y, z)$ that has its origin $O$ in the center of the LMC, whose coordinates are $\left(\alpha_{0}, \delta_{0}, D_{0}\right)$, with the $x$-axis antiparallel to the right ascension axis, the $y$-axis 
parallel to the declination axis, and the $z$-axis pointing towards the observer. To describe the internal kinematics of the galaxy it is opportune to introduce a second cartesian coordinate system $\left(x^{\prime}, y^{\prime}, z^{\prime}\right)$ that is obtained from the system $(x, y, z)$ by a counterclockwise rotation around the $z$-axis by an angle equal to the position angle of the line of nodes $\Theta-\frac{\pi}{2}$, followed by a rotation around the new $x^{\prime}$-axis by an angle equal to the inclination angle of the disk plane $i$. With this definition the $\left(x^{\prime}, y^{\prime}\right)$ plane coincides with the equatorial plane of the LMC galaxy, and the $x^{\prime}$-axis is along the line of nodes.

\subsection{LMC disk}

Following the new results on the LMC morphology discussed in Sect. 2, we consider an elliptical isothermal flared disk tipped by an angle $i=34.7^{\circ} \pm 6.2^{\circ}$ with respect to the sky plane, with the closest part in the north-east side. The center of the disk coincides with the center of the bar located at $\mathrm{J} 2000(\alpha, \delta)=\left(5^{\mathrm{h}} 27.6^{\mathrm{m}} \pm 3.9^{\mathrm{m}},-69.87^{\circ} \pm 0.41^{\circ}\right)$ and its distance from us is $D_{0}=50.1 \pm 2.5 \mathrm{kpc}$. Here we take a bar mass $M_{\text {bar }}=1 / 5 M_{\text {disk }}($ Sahu 1994; Gyuk et al. 2000), with the visible mass in disk and bar $M_{\text {bar }}+M_{\text {disk }}=(2.7 \pm 0.6) \times 10^{9} M_{\odot}$ (van der Marel et al. 2002).

The vertical distribution of stars in an isothermal disk is described by the $\operatorname{sech}^{2}$ function, therefore the spatial density of the disk is modelled by:

$\rho_{\mathrm{d}}=\frac{\mathcal{N} M_{\mathrm{d}}}{4 \pi q R_{\mathrm{d}}^{2} \zeta_{\mathrm{d}}(0)} \operatorname{sech}^{2}\left(\frac{\zeta}{\zeta_{\mathrm{d}}(R)}\right) \mathrm{e}^{-\frac{1}{R_{\mathrm{d}}} \sqrt{\left(\frac{\xi}{q}\right)^{2}+\eta^{2}}}$,

where $q=0.688$ is the ellipticity factor, $R_{\mathrm{d}}=1.54 \mathrm{kpc}$ is the scale length of the exponential disk, $R$ is the radial distance from the center on the disk plane. $\zeta_{\mathrm{d}}(R)$ is the flaring scale height, which rises from $0.27 \mathrm{kpc}$ to $1.5 \mathrm{kpc}$ at a distance of $5.5 \mathrm{kpc}$ from the center (van der Marel et al. 2002), and is given by

$\zeta_{\mathrm{d}}(R)=0.27+1.40 \tanh \left(\frac{R}{4}\right)$.

$\mathcal{N}=0.2765$ is a normalization factor that takes into account the flaring scale height.

The Cartesian coordinates $\left(\xi, \eta, \zeta \equiv z^{\prime}\right)$ are obtained from the system $\left(x^{\prime}, y^{\prime}, z^{\prime}\right)$ by rotation around the $z^{\prime}$-axis by an angle equal to $\left(\Phi-\Theta-\frac{\pi}{2}\right)$, where $\Phi$ is the position angle of the LMC disk major axis. In this way the $\xi-\eta$ plane coincides with the equatorial disk plane and the $\eta$ axis is directed along the major axis of the elliptic disk.

The velocity dispersion is a crucial parameter for the estimate of the microlensing rate. The kinematic studies of the LMC disk have shown that measurements of the velocity dispersion along the line of sight vary between roughly 6 and $30 \mathrm{~km} \mathrm{~s}^{-1}$, according to the age of the tracers, and show little variation as a function of the radius (see Gyuk et al. 2000, where a comprehensive table is given). In particular, younger populations have a smaller velocity dispersion than older ones, as in the MW. In the picture of van der Marel et al. (2002) within a distance of about $3 \mathrm{kpc}$ from the center of the LMC, the line of sight velocity dispersion (evaluated for carbon stars) can be considered as constant with $\sigma_{\mathrm{d}, \mathrm{los}}=20.2 \pm 0.5 \mathrm{~km} \mathrm{~s}^{-1}$.

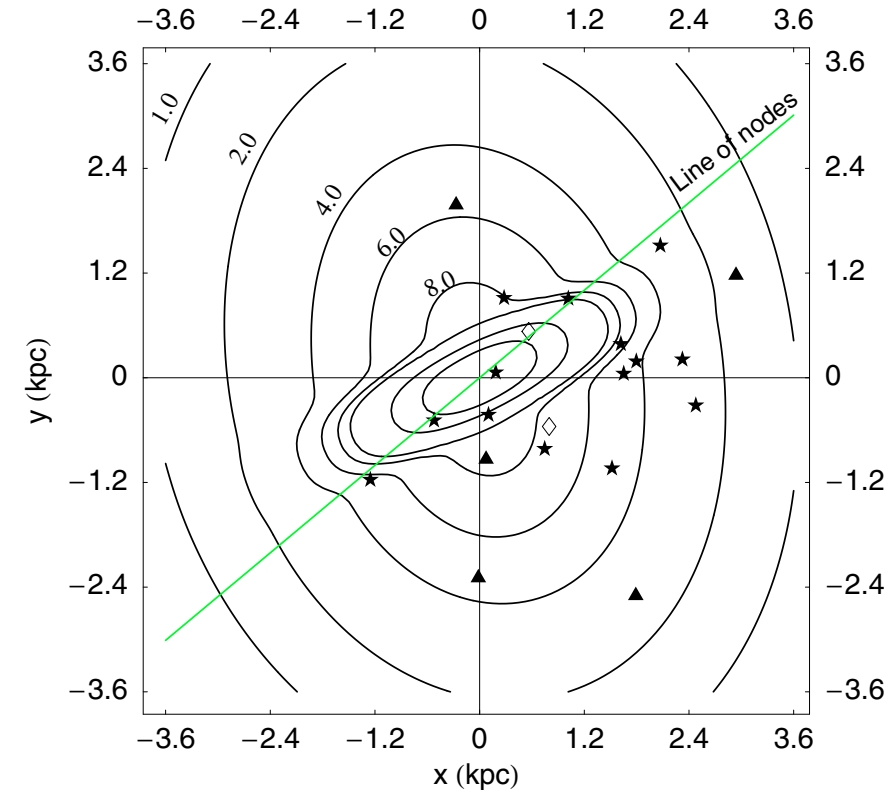

Fig. 1. Projection on the sky plane ( $x-y$ plane) of the column density of the LMC disk and bar. The numerical values on the contours are in $10^{7} M_{\odot} \mathrm{kpc}^{-2}$ units. The three innermost contours correspond to 10 , 20 and $30 \times 10^{7} M_{\odot} \mathrm{kpc}^{-2}$. The locations of the MACHO (black stars and empty diamonds) and EROS (triangles) microlensing candidates are also shown.

This represents our choice for the line of sight velocity dispersion of the disk stars.

\section{2. $L M C$ bar}

An optical bar of roughly $3^{\circ} \times 1^{\circ}$ angular size stands out in the central region of the LMC. Unlike the disk, the geometry of the bar region, in particular its vertical structure, is not well constrained. Understanding how the bar is displaced with respect to the disk is one of the last bits of information needed to complete the puzzle of the LMC morphology.

In Paper I we have described the bar by a Gaussian density profile following Gyuk et al. (2000). In this paper we choose, instead, a bar spatial density that takes into account its boxy shape, as in Zhao \& Evans (2000):

$\rho_{\mathrm{b}}=\frac{2 M_{\mathrm{b}}}{\pi^{2} R_{\mathrm{b}}^{2} \Xi_{\mathrm{b}}} \mathrm{e}^{-\left(\frac{\bar{\Xi}_{\mathrm{b}}}{\overline{\mathrm{b}}_{\mathrm{b}}}\right)^{2}} \mathrm{e}^{-\frac{1}{R_{\mathrm{b}}^{4}}\left(\Upsilon^{2}+\zeta^{2}\right)^{2}}$,

where $\Xi_{\mathrm{b}}=1.2 \mathrm{kpc}$ is the scale length of the bar axis, $R_{\mathrm{b}}=$ $0.44 \mathrm{kpc}$ is the scale height along a circular section, and the cartesian coordinates $\left(\Xi, \Upsilon, \zeta \equiv z^{\prime}\right)$ are obtained from the system $\left(x^{\prime}, y^{\prime}, z^{\prime}\right)$ by rotating around the $z^{\prime}$-axis by an angle equal to $(\Psi-\Theta)$, where $\Psi$ is the position angle of the LMC bar major axis, roughly $\simeq 120^{\circ}$ (van der Marel 2001). We fix it to $112.7^{\circ}$, so that the bar major axis $\Xi$ coincides with the minor axis of the disk $\xi$.

The column density, projected on the $x-y$ plane, is plotted in Fig. 1, giving a global view of the LMC shape (disk and bar coplanar) for a terrestrial observer. We indicate the direction of the line of nodes, together with the positions of the microlensing events detected by the MACHO (filled stars and empty 
diamonds) and EROS (filled triangles) ${ }^{2}$ collaborations. The maximum value of the column density, $41.5 \times 10^{7} M_{\odot} \mathrm{kpc}^{-2}$, is assumed in the center of the LMC. The positions of the MACHO microlensing events in this $x-y$ reference system are reported in Table 1.

In order to explore the consequences of different bar geometries, besides the coplanar configuration as in Gyuk et al. (2000) and Weinberg \& Nikolaev (2001), we have considered two other possible geometries. In particular, we drop the hypothesis that the disk and bar components are dynamically connected. In a first case, drawing inspiration from the paper of Zhao \& Evans (2000), we rotate the bar around an axis through the center, orthogonal to the plane defined by the bar axis and the line of sight. As a second case we shift the bar towards the observer along the line of sight as in Nikolaev et al. (2004). In both cases we change accordingly the scale parameters of the bar so as to keep its observed projected shape on the sky plane fixed. For both configurations we considered several values for the shift and the rotation angle in order to study their influence on the microlensing quantities. In the following, as an illustration, we will give the results for two somewhat extreme cases, so that we will get upper limits for the corresponding microlensing quantities

- a translation towards the observer of $\sim 0.5 \mathrm{kpc}$;

- a rotation of $40^{\circ}$ with the south-east side towards the observer. In this case the linearly growing distance between bar and disk is $\sim 1.0 \mathrm{kpc}$ at one scale length from the center.

For the velocity dispersion of the stellar population of the bar, for which very few data are available, we take into account the qualitative results from numerical simulation that show a general trend of a higher line of sight velocity dispersion in the central region of the LMC (Zasov \& Khoperskov 2002). We consider again two somewhat extreme cases: a line of sight velocity dispersion equal to that of the disk, $\sigma_{\mathrm{b}, \mathrm{los}}=20.2 \mathrm{~km} \mathrm{~s}^{-1}$, and a second case with a higher value, $\sigma_{\mathrm{b}, \mathrm{los}}=30 \mathrm{~km} \mathrm{~s}^{-1}$.

\section{3. $L M C$ halo}

We use two different models to describe the halo profile density: a spherical halo (model S) and an ellipsoidal halo (model E). The values of the parameters have been chosen so that the models have roughly the same mass within the same radius.

- Model S. In this model we neglect the tidal effects due to our Galaxy, and we adopt a classical pseudo-isothermal spherical density profile, which allows us to compare the results obtained in this paper with the previous ones:

$$
\rho_{\mathrm{h}, \mathrm{S}}=\rho_{0, \mathrm{~S}}\left[1+\frac{R^{2}}{a^{2}}\right]^{-1} \theta\left(R_{\mathrm{t}}-R\right),
$$

where $a$ is the LMC halo core radius, $\rho_{0, \mathrm{~S}}$ the central density, $R_{\mathrm{t}}$ a cutoff radius and $\theta$ the Heaviside step function.

\footnotetext{
2 The EROS group has published the detection of 5 microlensing events towards the LMC (Lasserre et al. 2000). The event EROS2LMC-5 is dubious as its light curve is asymmetric. This candidate has actually been considered by MACHO as its candidate LMC-26 and rejected as being a supernova (Milsztajn 2003).
}

We use $a=2 \mathrm{kpc}$ (Alcock et al. 2000a). We fix the value for the mass of the halo within a radius of $8.9 \mathrm{kpc}$ equal to $5.5 \times 10^{9} M_{\odot}$ (van der Marel et al. 2002) that implies $\rho_{0, \mathrm{~S}}$ equal to $1.76 \times 10^{7} M_{\odot} \mathrm{kpc}^{-3}$. Assuming a halo truncation radius, $R_{\mathrm{t}}=15 \mathrm{kpc}$ (van der Marel et al. 2002), the total mass of the halo is $\approx 1.08 \times 10^{10} M_{\odot}$.

- Model E. In this model the MW tidal effects induce an oblate spheroidal shape in the halo. We describe the halo density profile as

$\rho_{\mathrm{h}, \mathrm{E}}=\rho_{0, \mathrm{E}}\left(1+\frac{1}{a^{2}}\left(\frac{\xi^{2}}{q_{1}^{2}}+\eta^{2}+\zeta^{2}\right)\right)^{-1}$,

with $a=2 \mathrm{kpc}$, and the truncation radius $R_{\mathrm{t}}=15 \mathrm{kpc}$. For a LMC dark mass equal to $5.5 \times 10^{9} M_{\odot}$ within a radius of $8.9 \mathrm{kpc}$, the central density is $2.20 \times 10^{7} M_{\odot} \mathrm{kpc}^{-3}$, so that the LMC dark mass within the truncation radius turns out to be $\approx 1.06 \times 10^{10} M_{\odot}$.

\subsection{Galactic halo}

We will consider for the Galactic halo a spherical model with density profile given by:

$\rho_{\mathrm{GH}}=\rho_{0} \frac{R_{\mathrm{C}}^{2}+R_{\mathrm{S}}^{2}}{R_{\mathrm{C}}^{2}+R^{2}}$,

where $R$ is the distance from the Galactic center, $R_{\mathrm{C}}=5.6 \mathrm{kpc}$ (Primack et al. 1988) is the core radius, $R_{\mathrm{S}}=8.5 \mathrm{kpc}$ is the distance of the sun from the Galactic center and $\rho_{0}=7.9 \times$ $10^{6} M_{\odot} \mathrm{kpc}^{-3}$ is the mass density in the solar neighbourhood.

\section{Calculation of microlensing parameters}

As outlined in the introduction, here and in the following sections we want to fully exploit all the consequences of the geometry of the LMC, with the aim to shed new light on the still puzzling nature and location of the lenses detected in the microlensing surveys. In particular we study here two microlensing quantities, the optical depth and the microlensing rate.

In our analysis, whenever we need to compare models and predictions with observational results, we are going to use those presented by the MACHO collaboration only. The main reason is that this team has provided a complete description of their microlensing detection efficiency.

From the total set of 17 MACHO microlensing events ${ }^{3}$ one must exclude the event LMC-22, which is very likely a supernova of long duration or an active galactic nucleus in a galaxy at redshift $z=0.23$ (Alcock et al. 2001c).

We have detailed information on LMC-5: it is due to a lens located in the Galactic disk. Indeed the lens proper motion has been observed with the HST (Alcock et al. 2001a) and the lens mass determined to be either $\simeq 0.04 M_{\odot}$ or in the range $0.095-0.13 M_{\odot}$, so that it is a true brown dwarf or a M $4-5 \mathrm{~V}$ spectral type low mass star. The other stars which have been

\footnotetext{
The MACHO team actually claims the detection of 13-17 events according to the chosen selection criteria. In the following we shall consider for our purposes the largest set.
} 
microlensed were also observed by the HST, but no other lenses have been detected. This result is also confirmed by the analysis made by von Hippel et al. (2003), by using optical and near infrared photometry on a subset of five lensing sources which are LMC main sequence stars or slightly evolved subgiants, (LMC-4, LMC-6, LMC-8, LMC-9, LMC-14). Their analysis rules out as lenses main sequence stars with masses $\geq 0.1 M_{\odot}$ for distances out to $4 \mathrm{kpc}$.

The events LMC-9 and LMC-14 are known to be due to lenses belonging to the LMC itself, i.e. to the bar, disk or halo component. The latter event has been recognized thanks to the double source (Alcock et al. 2001b), while the first shows the characteristic caustic crossing signature of a double lens (Alcock et al. 2000b). For this reason we exclude the event LMC-9 from the following analysis, because we are interested in the study of a homogeneous set of single lens events.

We remark that LMC -5 , LMC -9 and LMC- 14 are the only events for which it has been possible to make a determination of the location of the lens. In Fig. 1 the events LMC-5 and LMC-9 are indicated with a special symbol, an empty diamond, whereas LMC-22 is not reported in the plot.

We use the interpolating function $\mathcal{E}(\hat{t})$ of the microlensing detection efficiency, calculated by the MACHO collaboration, as a function of event timescale $\hat{t}$ in the interval 1-1000 days (Fig. 5 of Alcock et al. 2000a). It is shown in Fig. 2 by the continuous line, together with some points of the MACHO efficiency, for comparison. Let us recall that the MACHO definition of the duration time $\hat{t}$ is twice the Einstein time $T_{\mathrm{E}}$, the parameter we use in this paper. We get the following expression for $\mathcal{E}(\hat{t})$ :

$$
\begin{aligned}
\mathcal{E}(\hat{t})= & f_{1}(\hat{t}) \theta[2.3-\log \hat{t}] \theta[\log \hat{t}] \\
& +f_{2}(\hat{t} \theta \theta \log \hat{t}-2.3] \theta[2.85-\log \hat{t}] \\
& +f_{3}(\hat{t}) \theta[\log \hat{t}-2.85] \theta[3-\log \hat{t}],
\end{aligned}
$$

with $\theta$ the Heaviside function, and

$$
\begin{aligned}
& f_{1}(\hat{t})=\frac{\log ^{3} \hat{t}}{\hat{t}^{1.97}}\left[8 \log ^{5} \hat{t}+\frac{49}{40} \log ^{8} \hat{t}\right] \\
& f_{2}(\hat{t})=\frac{f_{1}\left(10^{2.3}\right)-\frac{125}{64}(\log \hat{t}-2.3)^{3}}{10^{(2.3-\log \hat{t})^{4}}} \\
& f_{3}(\hat{t})=0.06+\left[f_{2}\left(10^{2.85}\right)-0.06\right] \mathrm{e}^{10(2.85-\log \hat{t}} .
\end{aligned}
$$

As described in the previous section, the LMC has its own halo, with a tidal radius $R_{\mathrm{t}}=15.0 \pm 4.5 \mathrm{kpc}$. Assuming that the Galactic halo extends to at least $65 \mathrm{kpc}$, a fraction of the LMC halo total mass, enclosed in a sphere of radius $R_{\mathrm{t}}$, is attributable to the Galactic halo. A simple calculation gives the Galactic halo mass enclosed in a sphere centered on the LMC with a radius of $8.9 \mathrm{kpc}$ to be $9.7 \times 10^{8} M_{\odot}$, a value corresponding to a sensible fraction of the LMC halo mass, of the order of $\simeq 17 \%$. We will take into account this fact, by properly correcting the value of the central density of the LMC halo: for the spherical model the value is decreased to $\rho_{0, \mathrm{~S}}=1.45 \times 10^{7} M_{\odot} \mathrm{kpc}^{-3}$ and the corresponding total LMC halo mass inside the tidal radius $R_{\mathrm{t}}$ is $\approx 8.86 \times 10^{9} M_{\odot}$; for the ellipsoidal model the value is decreased to $\rho_{0, \mathrm{E}}=1.81 \times 10^{7} M_{\odot} \mathrm{kpc}^{-3}$ and the corresponding total LMC halo mass inside the tidal radius $R_{\mathrm{t}}$ is $\approx 8.7 \times 10^{9} M_{\odot}$.

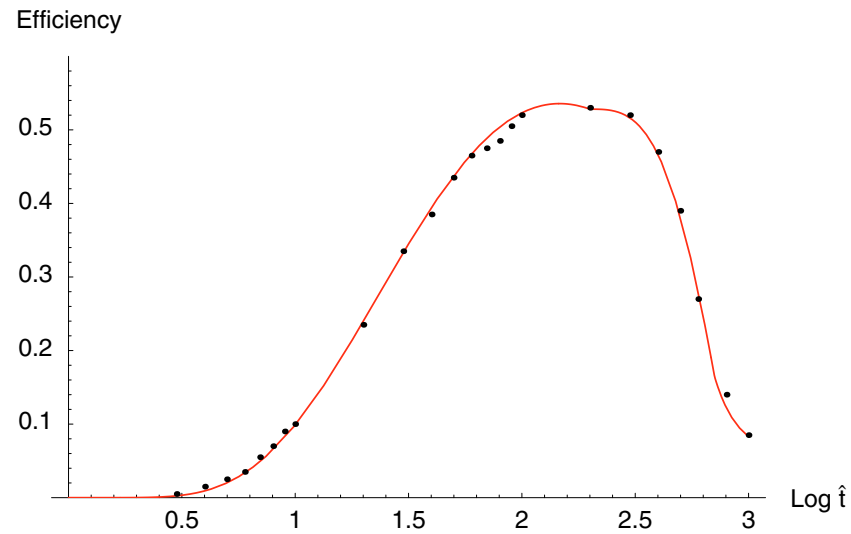

Fig. 2. Plot of the interpolating function $\mathcal{E}(\hat{t})$ of the MACHO detection efficiency.

\subsection{Optical depth}

We now discuss the results obtained for the optical depth. The computation is made by weighting the optical depth with respect to the distribution of the source stars along the line of view. We use Eq. (7), Sect. 3.1, of Paper I, that we report here for self-consistency reasons:

$\tau=\frac{4 \pi G}{c^{2}} \frac{\int_{0}^{\infty}\left[\int_{0}^{D_{\mathrm{os}}} \frac{D_{\mathrm{ol}}\left(D_{\mathrm{os}}-D_{\mathrm{ol}}\right)}{D_{\mathrm{os}}} \rho_{\mathrm{l}} \mathrm{d} D_{\mathrm{ol}}\right] \rho_{\mathrm{s}} \mathrm{d} D_{\mathrm{os}}}{\int_{0}^{\infty} \rho_{\mathrm{s}} \mathrm{d} D_{\mathrm{os}}}$,

where $\rho_{1}$ denotes the mass density of the lenses, $\rho_{\mathrm{s}}$ the mass density of the sources, $D_{\mathrm{ol}}$ and $D_{\mathrm{os}}$, respectively, the distance observer-lens and observer-source.

The contour maps reported in Figs. 3-6 refer to the case of coplanarity between bar and disk. Figure 3 shows the optical depth contour map for lenses in the Galactic halo, on the hypothesis that the whole Galactic dark halo consists of compact lenses, together with the positions of the MACHO fields (see Fig. 14 for the numeration of the fields), the microlensing events and the van der Marel line of nodes. We observe that almost all the fields (except three) fall between the contour lines corresponding to $\tau=46.3 \times 10^{-8}$ and $\tau=49.3 \times 10^{-8}$. As expected, the optical depth due to the Galactic halo is a slowly variable function, and presents a slight near-far asymmetry: moving from the nearer to the farther fields along a line passing through the center and perpendicular to the line of nodes, the increase of the optical depth is of the order of $\approx 6 \%$.

In Figs. 4 and 5 we report the optical depth contour maps for lenses belonging to the halo of the LMC, assuming a spherical and an ellipsoidal shape, respectively, on the hypothesis that the whole LMC dark halo consists of compact lenses. A striking feature of both maps is the strong near-far asymmetry.

For Model S, the maximum value of the optical depth, $\tau_{\max , \mathrm{S}} \simeq 8.05 \times 10^{-8}$, is assumed in a point falling in field number 13, belonging to the fourth quadrant, at a distance of $\simeq 1.27 \mathrm{kpc}$ from the center. The value in the point symmetrical with respect to the center, belonging to the second quadrant and falling about at the upward left corner of field 82 , is $\tau_{\mathrm{S}} \simeq 4.30 \times 10^{-8}$. The increment of the optical depth is of the order of $\approx 87 \%$, moving from the nearer to the farther fields. 


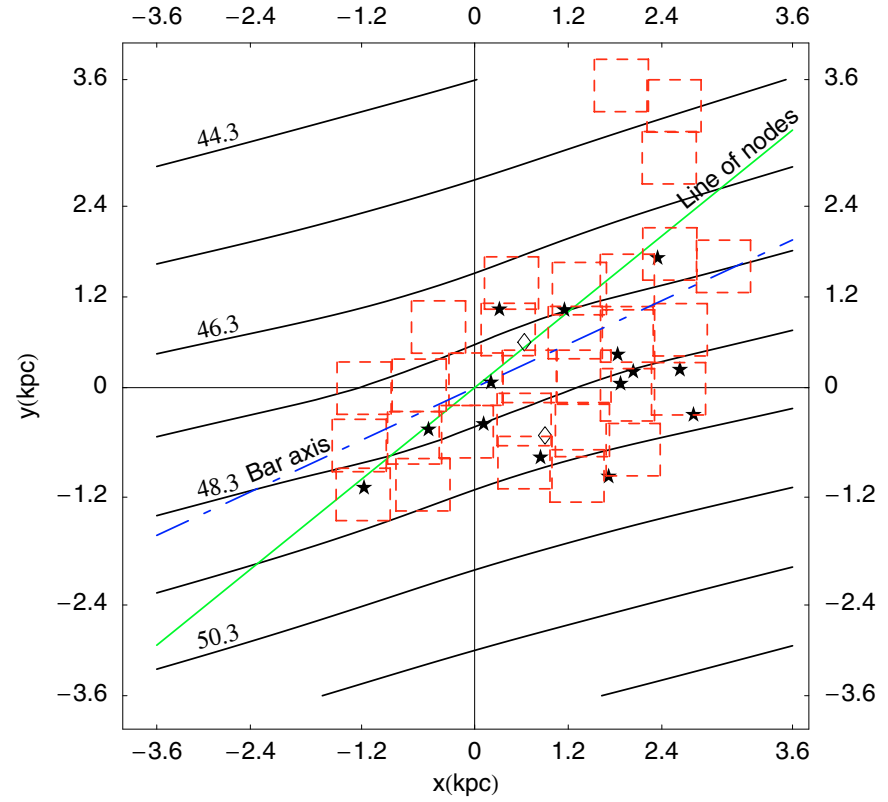

Fig. 3. Contour map of the optical depth for lenses in the Galactic halo. The locations of the MACHO fields and of the microlensing candidates are also shown. The numerical values are in $10^{-8}$ units.

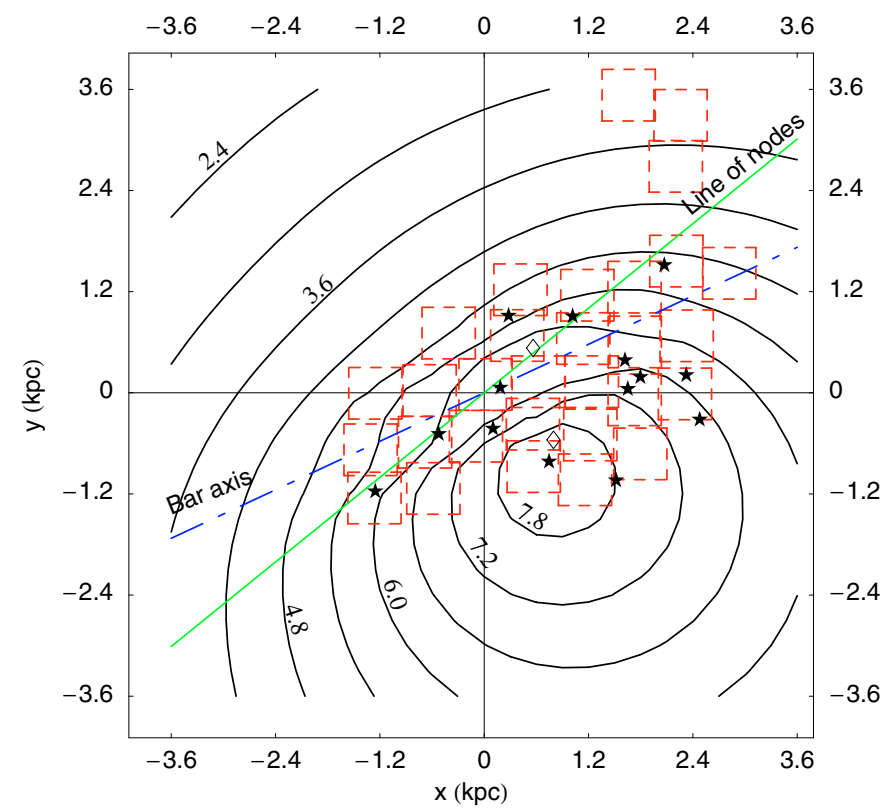

Fig. 4. Model S: contour map of the optical depth for lenses in the LMC halo. The locations of the MACHO fields and of the microlensing candidates are also shown. The numerical values are in $10^{-8}$ units.

The same is true for model $\mathrm{E}$ : the maximum value of the optical depth, $\tau_{\max , \mathrm{E}} \simeq 9.88 \times 10^{-8}$, higher than the previous one, is found at about the same point, at the same distance from the center. In the symmetrical point with respect to the center, belonging to field 82 , the optical depth is $\tau_{\mathrm{E}} \simeq 5.05 \times 10^{-8}$. The ellipsoidal shape of the LMC halo gives rise to a further enhancement of the near-far asymmetry, with an increase of the optical depth by $\approx 95 \%$.

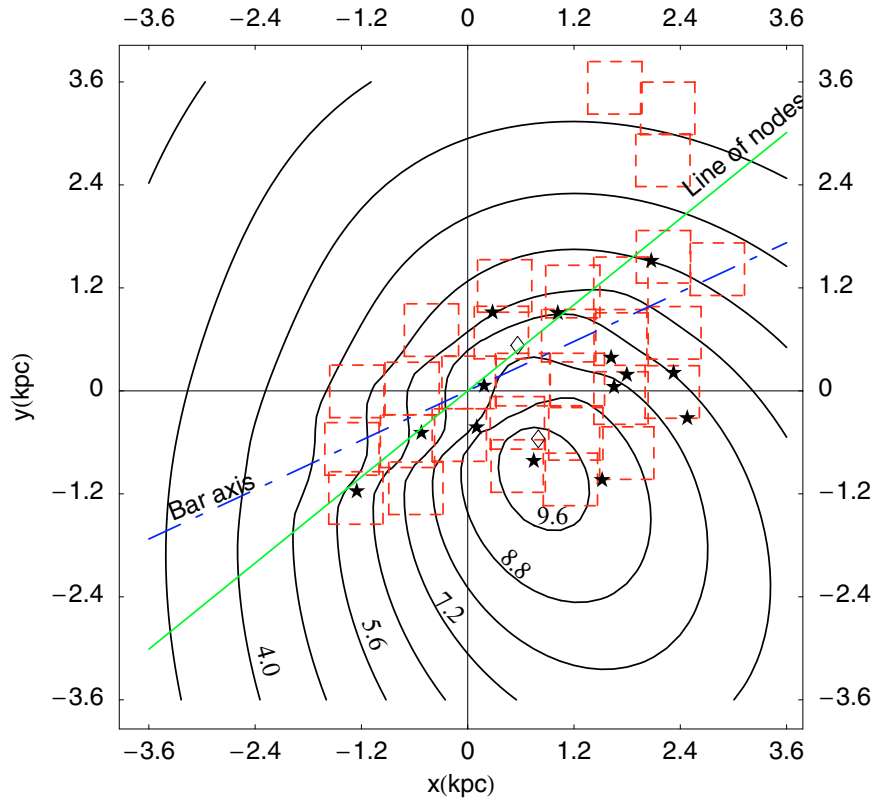

Fig. 5. Model E: contour map of the optical depth for lenses in the LMC halo. The locations of the MACHO fields and of the microlensing candidates are also shown. The numerical values are in $10^{-8}$ units.

One can draw advantage from the different asymmetric behaviour of the optical depth in the two cases of lenses in the Galactic halo or in the LMC halo, both to confirm the existence of a proper LMC halo and to disentangle the microlensing events due to the Galactic halo from the ones due to the LMC halo. To this end, a good observation strategy would help, the goal being to allow the analysis of asymmetry of microlensing events belonging to two equivalent regions, placed symmetrically with respect to the line of nodes. An example of the kind of analysis and of results one could obtain is given in Sect. 5.

In Fig. 6 we report the optical depth contour map for selflensing, i. e. for events where both the sources and the lenses belong to the stellar population of the disk and/or the bar of the LMC. As expected, there is almost ${ }^{4}$ no near-far asymmetry and the maximum value of the optical depth, $\tau_{\max } \simeq 4.90 \times$ $10^{-8}$, is reached in the center of the LMC. The optical depth then rapidly decreases, when moving, for instance, along a line going through the center and perpendicular to the minor axis of the elliptical disk, which coincides also with the major axis of the bar. In a range of about only $0.80 \mathrm{kpc}$ the optical depth quickly falls to $\tau \simeq 2 \times 10^{-8}$, and afterwards it decreases slowly to lower values.

The calculated value of the optical depth in the center seems at first glance to be in contradiction with the value one gets using the Gould inequality (Gould 1995):

$\tau \leq 2 \frac{\left\langle v^{2}\right\rangle}{c^{2}} \sec ^{2} i \approx 1.34 \times 10^{-8}$.

In fact, this inequality is derived making some simplifying assumptions which do not fully apply to our choice of the density profiles for the disk and the bar. In Sect. 6 we shall derive an

\footnotetext{
${ }^{4}$ We note the peculiar "ear" shape of the contour line for $\tau=2 \times$ $10^{-8}$ due to the disk flaring.
} 


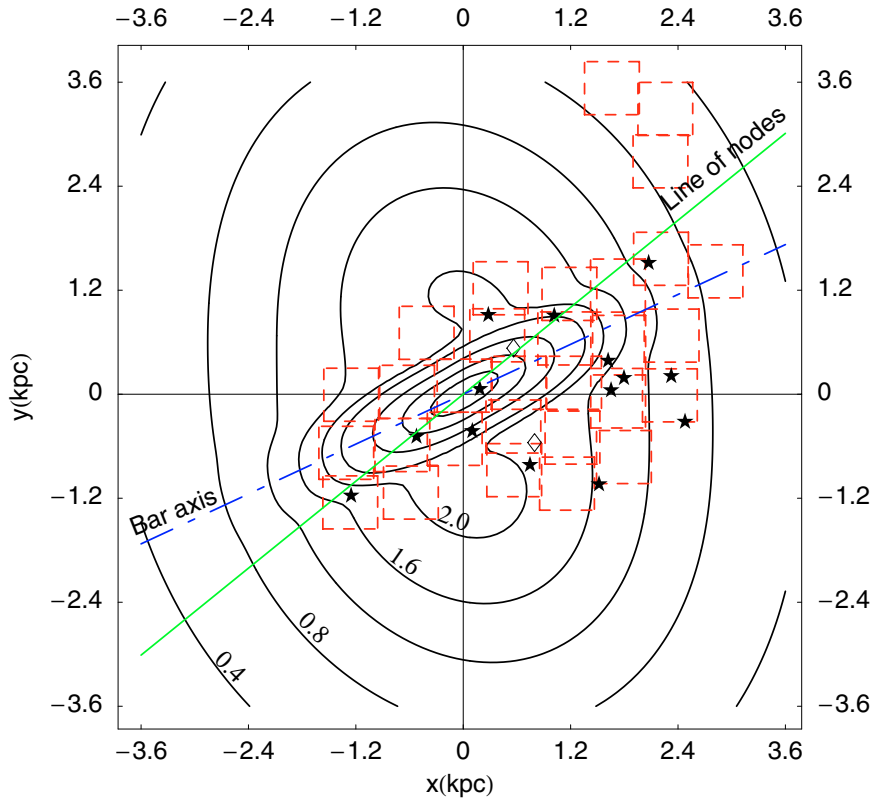

Fig. 6. Contour map of the optical depth for self-lensing. The locations of the MACHO fields and of the microlensing candidates are also shown. The numerical values are in $10^{-8}$ units. The innermost contours correspond to values $2.4 \times 10^{-8}, 3.2 \times 10^{-8}, 4.0 \times 10^{-8}$ and $4.6 \times 10^{-8}$ respectively.

improved version of the Gould inequality, which will also apply to our density profile and leads to $\tau \leq 6 \times 10^{-8}$, which does not contradict our above estimated value.

Furthermore, we recall that the Gould inequality is obtained under the hypothesis that the LMC is a virialized system, which quite likely is not the case for some of its components. This should of course be taken into account when using it in comparison with the observations.

As expected, the changes of the geometry as discussed in Sect. 3.2, where we allow for a non-coplanar morphology of the bar with respect to the disk, enhance the self-lensing optical depth in the bar region considerably (up to $\sim 50 \%$ ). On the other hand, the changes for the optical depth for lenses in the MW halo are negligible (at maximum $\sim 1 \%$ ). For lenses in the LMC halo the variations, in the innermost region of the $\mathrm{LMC}$, can be rather large (up to $\sim 20 \%$ ). However, the main feature that interests us, the near-far asymmetry due to the disk inclination, is not altered. Besides, as we are here mainly concerned with the self-lensing case, we do not discuss them any further. In any case, outside the bar region, these differences drop abruptly to zero.

For a rotated bar, Fig. 7, the increment of the self-lensing optical depth, with respect to the symmetric coplanar case, is slightly asymmetric with respect to the bar major axis. This is due to the different variations of the source-lens distances between the west side, where the sources are in the bar and the lenses in the disk, and the east side, where the opposite happens. The relative increment can be as large as $\approx 50 \%$.

When we allow for a translation of the bar, Fig. 8, the variation of the optical depth, with respect to the coplanar case, is higher in the region below the bar major axis (south-west side),

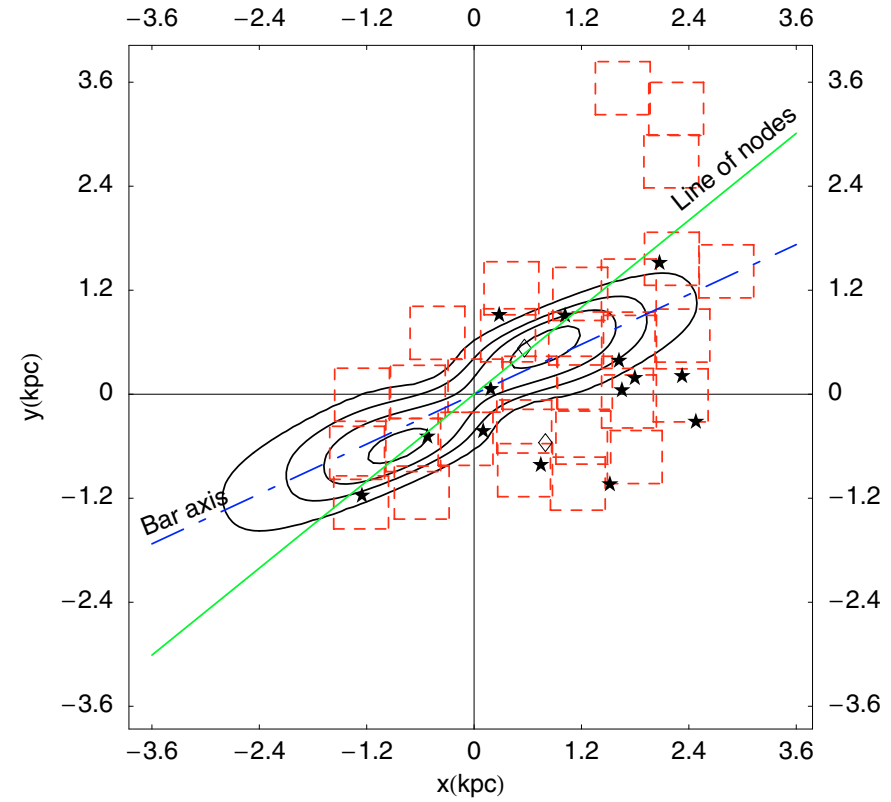

Fig. 7. Contour map of the difference between the self-lensing optical depth calculated in the case of $+40^{\circ}$ rotated bar and the one with coplanar bar and disk. Going from the outer to the inner part, the contours correspond to increasing values of the difference: $0.1,0.6,1.1$ and $1.6 \times 10^{-8}$. The difference is null outside the bar region.

even if the projected density of the bar is the same as shown in Fig. 1. This is induced by the inclination of the disk plane, which gives rise to increasing distances between the bar and the disk plane for lines of sight towards the lower part of the bar. In this case we also find a small region with a negative difference of the optical depth, shown by the lighter contour lines (northeast side). The relative increment can be as large as $\approx 35 \%$.

It is interesting to note that a larger statistics of observed events in the central region might eventually allow one to discriminate between the different bar models.

We notice also that, due to the loss of the symmetry, the Gould inequality no longer applies in these cases.

\subsection{Self-lensing microlensing event rate}

The distribution $\frac{\mathrm{d} \Gamma}{\mathrm{d} T_{\mathrm{E}}}$, the differential rate of microlensing events with respect to the Einstein time $T_{\mathrm{E}}$, allows one to estimate the expected typical duration and the expected number of the microlensing events. In this paper we evaluate the microlensing rate in the self-lensing configuration, i.e. lenses and sources both in the disk and/or in the bar of the LMC. We also take into account the transverse motion of the Sun and of the source stars.

We assume that, to an observer comoving with the LMC center, the velocity distribution of the source stars and lenses has a Maxwellian profile ${ }^{5}$. Moreover, since the component

5 The Maxwellian profile of the velocity distribution is the first term of a series expansion in terms of Gauss-Hermite moments (van der Marel \& Franx 1993; Gerhard 1993). See Sect. 3.2 of Paper I. 


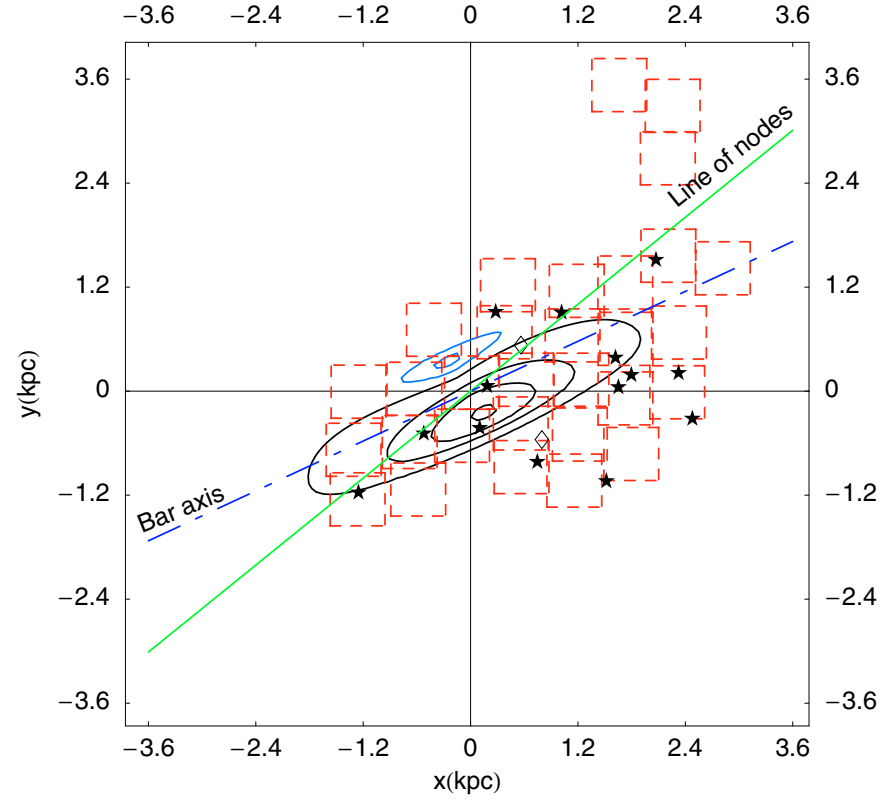

Fig. 8. Contour map of the difference between the self-lensing optical depth calculated in the case of a shift of $0.5 \mathrm{kpc}$ of the bar and the one with coplanar bar and disk. Going from the outer to the inner part, the black contours correspond to increasing positive values of the difference: $+0.1,+0.5,+0.9$, and $+1.3 \times 10^{-8}$; the lighter contours to negative values of the difference: -0.1 , and $-0.2 \times 10^{-8}$.

parallel to the line of view is irrelevant for microlensing, we integrate over the parallel component. Therefore, the twodimensional transverse velocity distribution of the source and lens stars is:

$f\left(\tilde{\boldsymbol{v}}_{\perp}\right) \mathrm{d}^{2} \tilde{v}_{\perp}=\frac{1}{2 \pi \sigma_{\text {los }}^{2}} \mathrm{e}^{\left(-\frac{\tilde{v}_{\perp}^{2}}{2 \sigma_{\mathrm{los}}^{2}}\right)} \tilde{v}_{\perp} \mathrm{d} \tilde{v}_{\perp} \mathrm{d} \varphi$

where we have used cylindrical coordinates ${ }^{6} \mathrm{~d}^{2} \tilde{v}_{\perp}=\tilde{v}_{\perp} \mathrm{d} \tilde{v}_{\perp} \mathrm{d} \phi$.

With respect to an observer comoving with the LMC center, the transverse velocity of the microlensing tube at position $D_{\mathrm{ol}}=x D_{\mathrm{os}}(0 \leq x \leq 1)$ is given by $\tilde{\boldsymbol{v}}_{\mathrm{t} \perp}=(1-x) \tilde{\boldsymbol{v}}_{\odot \perp}+x \tilde{\boldsymbol{v}}_{\mathrm{s} \perp}$, where $\tilde{\boldsymbol{v}}_{\mathrm{S} \perp}$ is the velocity of the source, and $\tilde{\boldsymbol{v}}_{\odot \perp}$ represents the velocity of the Sun in the plane orthogonal to the line of sight, as measured by an observer comoving with the LMC center. It results $\left|\tilde{\boldsymbol{v}}_{\odot \perp}\right| \simeq 406 \mathrm{~km} \mathrm{~s}^{-1}$ (van der Marel et al. 2002).

Let us consider a segment of a cylindrical ring at position $x$, of length $\mathrm{d} D_{\text {ol }}$, with radius equal to the Einstein radius

$R_{\mathrm{E}}=\left(\frac{4 G M_{\odot}}{c^{2}} \mu D_{\mathrm{os}} x(1-x)\right)^{\frac{1}{2}}$,

angular amplitude $\mathrm{d} \alpha$ and thickness $\left(\hat{\boldsymbol{v}}_{1 \perp} \cdot \hat{n}\right) \mathrm{d} t=\hat{v}_{1 \perp} \cos \theta \mathrm{d} t$, where $-\frac{\pi}{2} \leq \theta \leq \frac{\pi}{2}$ is the angle between the inner normal to the cylindrical ring segment and the vector $\hat{\boldsymbol{v}}_{1 \perp}$. $\hat{\boldsymbol{v}}_{1 \perp}$ represents the lens velocity in the plane orthogonal to the line of sight, as measured by an observer comoving with the microlensing tube at position $x$. We use solar mass units, defining $\mu=\frac{M}{M_{\odot}}$,

\footnotetext{
${ }^{6}$ Equation (12) gives the correct mean square transverse velocity $\left\langle\tilde{v}_{\perp}^{2}\right\rangle=2 \sigma_{\text {los }}^{2}$.
}

where $M$ is the lens mass. The number of lenses with mass in the range $(\mu, \mu+\mathrm{d} \mu)$ inside the volume element of the cylindrical ring segment is equal to the product of the differential number density of the lenses $\frac{\mathrm{d} n_{1}}{\mathrm{~d} \mu} \mathrm{d} \mu$ and the volume element $\hat{v}_{1 \perp} \cos \theta R_{\mathrm{E}} \mathrm{d} t \mathrm{~d} \alpha \mathrm{d} D_{\mathrm{ol}}$. The fraction entering the microlensing tube in the time interval $\mathrm{d} t$ with transverse velocity $\hat{\boldsymbol{v}}_{1 \perp}$, is given by those lenses that with respect to the LMC reference frame have velocity

$\tilde{\boldsymbol{v}}_{1 \perp}=\hat{\boldsymbol{v}}_{1 \perp}+\tilde{\boldsymbol{v}}_{\mathrm{t} \perp}=\hat{\boldsymbol{v}}_{1 \perp}+(1-x) \tilde{\boldsymbol{v}}_{\odot \perp}+x \tilde{\boldsymbol{v}}_{\mathrm{S} \perp}$.

In the self-lensing case, $x \simeq 1$, and to first approximation we can neglect the term proportional to $(1-x)$. As a consequence the velocity distribution with respect to the microlensing tube, in the general case of two different values for the velocity dispersion, is given by:

$$
\begin{gathered}
\frac{\hat{v}_{1 \perp} \mathrm{d} \hat{v}_{1 \perp} \mathrm{d} \theta}{2 \pi\left[\rho_{\mathrm{d}}(x)+\rho_{\mathrm{b}}(x)\right]}\left[\mathrm{e}^{\left(-\frac{\hat{v}_{\perp \perp}^{2}+2 x \hat{v}_{1 \perp} \tilde{v}_{\mathrm{s} \perp} \cos (\alpha-\theta)+x^{2} \tilde{\mathrm{s}}_{\mathrm{s}}^{2}}{2 \sigma_{\mathrm{d}}^{2}}\right)}\right. \\
\left.\times \frac{\rho_{\mathrm{d}}(x)}{\sigma_{\mathrm{d}}^{2}}+\mathrm{e}^{\left(-\frac{\hat{v}_{\perp \perp}^{2}+2 x \hat{v}_{1 \perp} \tilde{v}_{\mathrm{s} \perp} \cos (\alpha-\theta)+x^{2} \tilde{v}_{\mathrm{s} \perp}^{2}}{2 \sigma_{\mathrm{b}}^{2}}\right)} \frac{\rho_{\mathrm{b}}(x)}{\sigma_{\mathrm{b}}^{2}}\right] \\
=\frac{\hat{v}_{1 \perp} \mathrm{d} \hat{v}_{1 \perp} \mathrm{d} \theta}{2 \pi\left[\rho_{\mathrm{d}}(x)+\rho_{\mathrm{b}}(x)\right]} h\left(\rho_{\mathrm{d}}, \sigma_{\mathrm{d}} ; \rho_{\mathrm{b}}, \sigma_{\mathrm{b}} ; x, \hat{v}_{1 \perp}, \tilde{v}_{\mathrm{s} \perp} ; \alpha, \theta\right)
\end{gathered}
$$

where $\sigma_{\mathrm{d}} \equiv \sigma_{\mathrm{d}, \text { los }}, \sigma_{\mathrm{b}} \equiv \sigma_{\mathrm{b}, \text { los }}$ and $\alpha$ is the angle that the segment of a cylindrical ring at position $x$, of angular amplitude $\mathrm{d} \alpha$, forms with the direction of the orthogonal component to the line of sight of the velocity vector of the source star, $\tilde{\boldsymbol{v}}_{\mathrm{S} \perp}$.

We need now to specify the form of the number density. Assuming that the mass distribution of the lenses is independent of their position in the LMC (factorization hypothesis de Rújula et al. 1995), the lens number density per unit mass is given by

$\frac{\mathrm{d} n_{1}}{\mathrm{~d} \mu}=\frac{\rho_{\mathrm{d}}+\rho_{\mathrm{b}}}{M_{\odot}} \frac{\mathrm{d} n_{0}}{\mathrm{~d} \mu}$,

where we use $\frac{\mathrm{d} n_{0}}{\mathrm{~d} \mu}$ as given in Chabrier (2001). We consider both the power law and the exponential initial mass functions ${ }^{7}$. However, we find that our results do not depend strongly on that choice, and hereafter we will discuss the results we obtain by using the exponential IMF only.

Let us note that, considering the experimental conditions for the observations of the MACHO team, we use as range of variability for the lens masses $0.08 \leq \mu \leq 1.5$. Namely the lower limit is fixed by the requirement that the lenses belong to the luminous component of the LMC, while the upper limit is fixed by the requirement that the lenses are not resolved stars ${ }^{8}$.

The total differential rate $\mathrm{d} \Gamma$ at which lenses enter the portion of the microlensing tube at position $x$, along a fixed line of

\footnotetext{
${ }^{7}$ We have used the same normalization as in Paper I with the mass varying in the range 0.08 to $10 M_{\odot}$.

8 We have checked that the results are insensitive to the precise upper limit value. This is also confirmed by the following analysis of the expected value for the mass of the lenses.
} 
sight, is given by (de Rújula et al. 1991; Griest 1991):

$$
\begin{aligned}
\mathrm{d} \Gamma= & \frac{\hat{v}_{1 \perp}^{2} \mathrm{~d} \hat{v}_{1 \perp}}{4 \pi^{2} \mathcal{N} M_{\odot}} \int_{0}^{2 \pi} \mathrm{d} \varphi \int_{-\frac{\pi}{2}}^{\frac{\pi}{2}} \mathrm{~d} \theta \cos \theta \\
& \times \int_{d_{\min }}^{d_{\max }} D_{\mathrm{os}} \mathrm{d} D_{\mathrm{os}} \int_{\frac{d_{\min }}{D_{\mathrm{os}}}}^{1} \mathrm{~d} x \int_{0.08}^{1.5} \mathrm{~d} \mu \frac{\mathrm{d} n_{0}}{\mathrm{~d} \mu} R_{\mathrm{E}} \\
& \times \int_{0}^{\infty} \mathrm{d} \tilde{v}_{\mathrm{s} \perp} \tilde{v}_{\mathrm{s} \perp}\left[\frac{\rho_{\mathrm{d}}\left(D_{\mathrm{os}}\right)}{\sigma_{\mathrm{d}}^{2}} \mathrm{e}^{-\frac{\tilde{v}_{\mathrm{s} \perp}^{2}}{2 \sigma_{\mathrm{d}}^{2}}}+\frac{\rho_{\mathrm{b}}\left(D_{\mathrm{os}}\right)}{\sigma_{\mathrm{b}}^{2}} \mathrm{e}^{-\frac{\tilde{v}_{\mathrm{s}}^{2}}{2 \sigma_{\mathrm{b}}^{2}}}\right] \\
& \times \int_{0}^{2 \pi} \mathrm{d} \alpha h\left(\rho_{\mathrm{d}}, \sigma_{\mathrm{d}} ; \rho_{\mathrm{b}}, \sigma_{\mathrm{b}} ; x, \hat{v}_{1 \perp}, \tilde{v}_{\mathrm{s} \perp} ; \alpha, \theta\right)
\end{aligned}
$$

where

$$
\mathcal{N}=\int_{d_{\min }}^{d_{\max }} \mathrm{d} D_{\mathrm{os}}\left[\rho_{\mathrm{d}}\left(D_{\mathrm{os}}\right)+\rho_{\mathrm{b}}\left(D_{\mathrm{os}}\right)\right],
$$

having assumed that the number of detectable stars varies with the distance as $D_{\mathrm{os}}^{-2}$. The integration limits $d_{\min }, d_{\mathrm{max}}$ represent the distances from the observer of the intersection points of the line of sight with the LMC tidal surface.

Finally, as we are interested in the distribution $\frac{\mathrm{d} \Gamma}{\mathrm{d} T_{\mathrm{E}}}$, we change variable from $\hat{v}_{1 \perp}$ to $T_{\mathrm{E}}$, bearing in mind that $\hat{v}_{1 \perp}=\frac{R_{\mathrm{E}}}{T_{\mathrm{E}}}$. After integration over $\varphi, \theta$ and $\alpha$, and taking into account the detection efficiency function, Eq. (6), we obtain:

$$
\begin{aligned}
\left(\frac{\mathrm{d} \Gamma}{\mathrm{d} T_{\mathrm{E}}}\right)_{\varepsilon}= & \frac{\mathrm{d} \Gamma}{\mathrm{d} T_{\mathrm{E}}} \cdot \mathcal{E}\left(T_{\mathrm{E}}\right)=\frac{32 G^{2} M_{\odot} \mathcal{E}\left(T_{\mathrm{E}}\right)}{c^{4} T_{\mathrm{E}}^{4} \mathcal{N}} \\
& \times \int_{d_{\min }}^{d_{\max }} \mathrm{d} D_{\mathrm{os}} D_{\mathrm{os}}^{3} \\
& \times \int_{\frac{d_{\min }}{D_{\mathrm{os}}}}^{1} \mathrm{~d} x x^{2}(1-x)^{2} \int_{0.08}^{1.5} \mathrm{~d} \mu \mu^{2} \frac{\mathrm{d} n_{0}}{\mathrm{~d} \mu} \\
& \times\left[\frac{\rho_{\mathrm{b}}(x) \rho_{\mathrm{b}}\left(D_{\mathrm{os}}\right)}{(1+x)^{2} \sigma_{\mathrm{b}}^{2}} \mathrm{e}^{\left(-\frac{2 G M_{\odot} D_{\mathrm{os}} x(1-x) \mu}{c^{2}\left(1+x^{2}\right) \sigma_{\mathrm{b}}^{2} T_{\mathrm{E}}^{2}}\right)}\right. \\
& +\frac{\rho_{\mathrm{d}}(x) \rho_{\mathrm{b}}\left(D_{\mathrm{os}}\right)}{\sigma_{\mathrm{d}}^{2}+x^{2} \sigma_{\mathrm{b}}^{2}} \mathrm{e}^{\left(-\frac{2 G M_{\odot} D_{\mathrm{os}} x(1-x) \mu}{c^{2}\left(\sigma_{\mathrm{d}}^{2}+x^{2} \sigma_{\mathrm{b}}^{2}\right) T_{\mathrm{E}}^{2}}\right)} \\
& +\frac{\rho_{\mathrm{b}}(x) \rho_{\mathrm{d}}\left(D_{\mathrm{os}}\right)}{\sigma_{\mathrm{b}}^{2}+x^{2} \sigma_{\mathrm{d}}^{2}} \mathrm{e}^{\left(-\frac{2 G M_{\odot} D_{\mathrm{os}} x(1-x) \mu}{c^{2}\left(\sigma_{\mathrm{b}}^{2}+x^{2} \sigma_{\mathrm{d}}^{2}\right) T_{\mathrm{E}}^{2}}\right)} \\
& \left.+\frac{\rho_{\mathrm{d}}(x) \rho_{\mathrm{d}}\left(D_{\mathrm{os}}\right)}{(1+x)^{2} \sigma_{\mathrm{d}}^{2}} \mathrm{e}^{\left(-\frac{2 G M_{\odot} D_{\mathrm{os}} x(1-x) \mu}{c^{2}\left(1+x^{2}\right) \sigma_{\mathrm{d}}^{2} T_{\mathrm{E}}^{2}}\right)}\right] .
\end{aligned}
$$

In the following section we need also the two distributions

$$
\begin{aligned}
& \left(\frac{\mathrm{d}^{2} \Gamma}{\mathrm{d} T_{\mathrm{E}} \mathrm{d} x}\right)_{\varepsilon}=\left.\mathcal{E}\left(T_{\mathrm{E}}\right) \frac{\mathrm{d}^{2} \Gamma}{\mathrm{d} T_{\mathrm{E}} \mathrm{d} x}\right|_{T_{\mathrm{E}, \text { event }}} \\
& \left(\frac{\mathrm{d}^{2} \Gamma}{\mathrm{d} T_{\mathrm{E}} \mathrm{d} \mu}\right)_{\varepsilon}=\left.\mathcal{E}\left(T_{\mathrm{E}}\right) \frac{\mathrm{d}^{2} \Gamma}{\mathrm{d} T_{\mathrm{E}} \mathrm{d} \mu}\right|_{T_{\mathrm{E}, \text { event }}}
\end{aligned}
$$

calculated assigning to $T_{\mathrm{E}}$ the corresponding effective measured value.

\subsection{A statistical analysis for self-lensing events discrimination}

In this section we will show that, in the framework of the LMC geometrical structure and dynamics outlined in Sect. 3, a suitable statistical analysis allows us to exclude from the self-lensing population a large subset of the detected events. To this purpose, assuming all the 15 events as self-lensing, we study the scatter plots correlating the self-lensing expected values of some meaningful microlensing variables with the measured Einstein time or with the self-lensing optical depth. The idea underlying this analysis is based on the search of selflensing average collective properties with different behaviour in the two different regions of the LMC: the bar with its nearby neighbourhood and the disk region external to it. In this way we eventually show that a large subset of events is incompatible with the self-lensing hypothesis.

We have calculated the self-lensing distributions $\left(\frac{\mathrm{d} \Gamma}{\mathrm{d} T_{\mathrm{E}}}\right)_{\varepsilon}$ of the rate of microlensing events with respect to the Einstein time $T_{\mathrm{E}}$, along the lines of sight towards the 15 events of the MACHO collaboration, in the case of a Chabrier exponential type IMF. As an example we show in Fig. 9 the distributions $\left(\frac{\mathrm{d} \Gamma}{\mathrm{d} T_{\mathrm{E}}}\right)_{\varepsilon}$ calculated along the lines of sight pointing towards the events LMC-1 (solid line), LMC-8 (dashed line) and LMC-23 (dot dashed line $)^{9}$.

With these distributions we have calculated the median ${ }^{10}$ $T_{\mathrm{E}, 50 \%}$ and the values $T_{\mathrm{E}, 16 \%}$ and $T_{\mathrm{E}, 84 \%}$ that single out the extremes of the $68 \%$ probability range around the median (not to be confused with a $1 \sigma$ error). In Table 1 we report these values for each observed MACHO event.

Trying to see if the geometry can help, we have analyzed how the self-lensing expected values of $T_{\mathrm{E}}$ depend on the position, or better still on the optical depth, taking into account that the LMC disk symmetry is elliptical and not circular.

In Fig. 10 we report on the $y$-axis the observed values of $T_{\mathrm{E}}$ (empty boxes) as well as the expected values for self-lensing of the median $T_{\mathrm{E}, 50 \%}$ (filled circle) evaluated along the directions of the events. On the $x$-axis we report the value of the selflensing optical depth calculated towards the event position. The optical depth increases as one moves from the outer regions towards the center of the LMC according to the contour lines shown in Fig. 6. An interesting feature emerging clearly is the decreasing trend of the expected values of the median $T_{\mathrm{E}, 50 \% \text {, }}$ going from the outside fields with low values of $\tau_{\mathrm{SL}}$ towards the central fields with higher values of $\tau_{\mathrm{SL}}$. The variation of the stellar number density and the flaring of the LMC disk certainly contributes to explaining this behaviour.

We now tentatively identify two subsets of events: the ten falling outside the contour line $\tau_{\mathrm{SL}}=2 \times 10^{-8}$ of Fig. 6 and the five falling inside. In the framework of van der Marel et al. LMC geometry, this contour line includes almost fully the

\footnotetext{
9 We have also used the Chabrier power law IMF, obtaining slightly higher values, of the order of $10 \%$, with respect to the exponential one. In the following we have used constantly the Chabrier exponential IMF.

10 As the distribution is strongly asymmetric, to describe the expected value of $T_{\mathrm{E}}$ we use the median value, a more meaningful parameter than the average value.
} 
Table 1. Microlensing rate: the results for the predicted Einstein time. Case with disk and bar coplanar and bar velocity dispersion $\sigma_{\mathrm{b}}=$ $20.2 \mathrm{~km} \mathrm{~s}^{-1}$. For each observed MACHO event we report for the Einstein time (days): the observed value and the predicted median value with the two extremes of a $68 \%$ probability range around it. In the second and third column we report the position in the reference frame centered in the LMC as defined in Sect. 3.

\begin{tabular}{ccccccc}
\hline \hline Event & $x(\mathrm{kpc})$ & $y(\mathrm{kpc})$ & $T_{\mathrm{E}, \mathrm{obs}}$ & $T_{\mathrm{E}, 50 \%}$ & $T_{\mathrm{E}, 16 \%}$ & $T_{\mathrm{E}, 84 \%}$ \\
\hline 1 & 1.017 & 0.909 & 22.3 & 64 & 33 & 126 \\
4 & 0.746 & -0.814 & 29.5 & 66 & 35 & 128 \\
5 & 0.797 & -0.559 & 49.1 & 65 & 34 & 127 \\
6 & 0.102 & -0.423 & 59.5 & 55 & 28 & 111 \\
7 & 1.796 & 0.189 & 66.8 & 73 & 38 & 140 \\
8 & 0.185 & 0.062 & 43.1 & 48 & 25 & 95 \\
13 & 0.280 & 0.916 & 65.0 & 66 & 33 & 128 \\
14 & -0.523 & -0.487 & 65.0 & 51 & 26 & 103 \\
15 & 1.652 & 0.048 & 23.9 & 72 & 38 & 137 \\
18 & -1.253 & -1.168 & 48.2 & 72 & 38 & 138 \\
20 & 2.478 & -0.316 & 47.2 & 77 & 40 & 146 \\
21 & 2.324 & 0.211 & 60.5 & 77 & 40 & 146 \\
23 & 1.517 & -1.037 & 55.4 & 72 & 38 & 138 \\
25 & 2.072 & 1.517 & 55.4 & 79 & 41 & 149 \\
27 & 1.619 & 0.388 & 32.8 & 67 & 34 & 130 \\
\hline
\end{tabular}

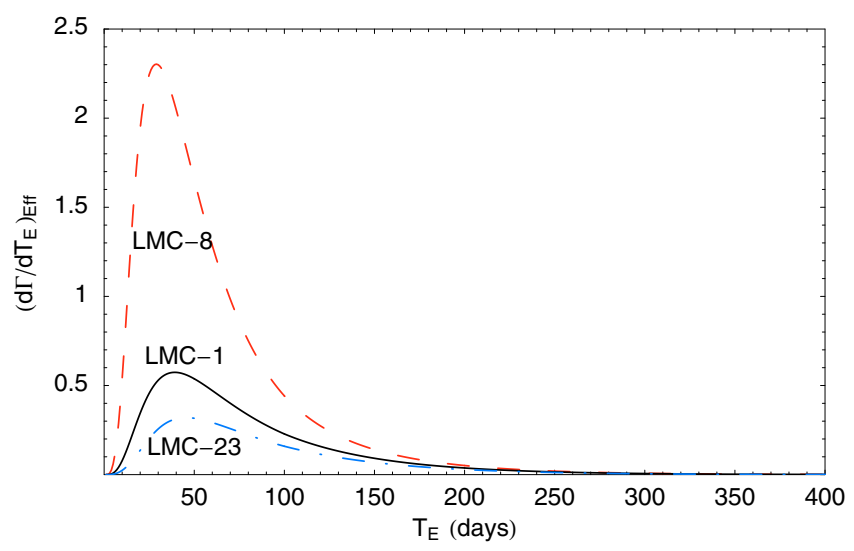

Fig. 9. Differential rate of the microlensing events with respect to the Einstein time $T_{\mathrm{E}}$, along the lines of sight pointing towards the events LMC-8 (dashed red line), LMC-1 (solid black line) and LMC-23 (dot dashed blue line). The $y$-axis values are in $10^{-12}$ units.

LMC bar and two ear shaped inner regions of the disk, where we expect self-lensing events to be located with higher probability.

At first glance, we note that the two clusters have a clear-cut different collective behaviour: the measured Einstein times of the first 10 points fluctuate around a median value of 47 days, very far from the expected values of the median $T_{\mathrm{E}}$, ranging from 65 days to 79 days, with an average value of 72 days. On the contrary, for the last 5 points, the measured Einstein times fluctuate around a median value of 51 days, near to the average value 57 days of the expected medians, ranging from 48 days to 66 days. Let us note, also, the somewhat peculiar position of the event $\mathrm{LMC}-1$, with a very low value of the observed $T_{\mathrm{E}}$. In the following analysis it will be shown that most probably

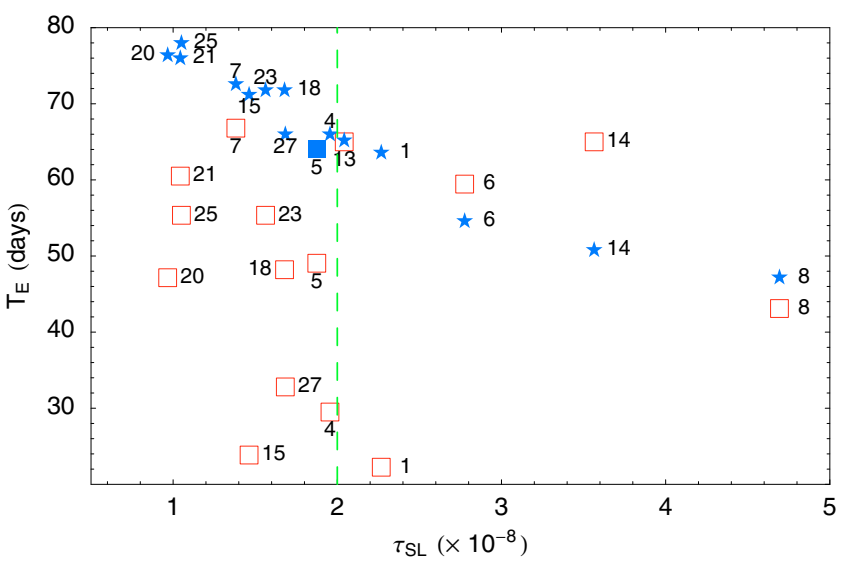

Fig. 10. Scatter plot of the observed (empty boxes) values of the Einstein time and of the expected values of the median $T_{\mathrm{E}, 50 \%}$ (filled stars), with respect to the self-lensing optical depth evaluated along the directions of the events. The $T_{\mathrm{E}, 50 \%}$ for the Galactic disk event LMC- 5 is represented by a filled box.

this event is homogeneous to the set at left to the vertical line in Fig. 10 and it has to be included in that cluster.

This plot gives a first clear evidence that, in the framework of van der Marel et al. LMC geometry, the self-lensing events have to be searched for among the cluster of events with $\tau_{\mathrm{SL}}>$ $2 \times 10^{-8}$, and at the same time that the cluster of the 10 events plus LMC-1 belongs, very probably, to a different population.

In order to further improve our statistical analysis, we have calculated the distributions $\left(\frac{\mathrm{d}^{2} \Gamma}{\mathrm{d} T_{\mathrm{E}} \mathrm{d} \mu}\right)_{\varepsilon}$ along the lines of sight pointing towards the $14 \mathrm{LMC}$ microlensing events ${ }^{11}$, taking, for each line of sight, the observed Einstein time value. As an

11 The previous 15 events minus the event LMC-5, formerly recognized as a Galactic disk event. 


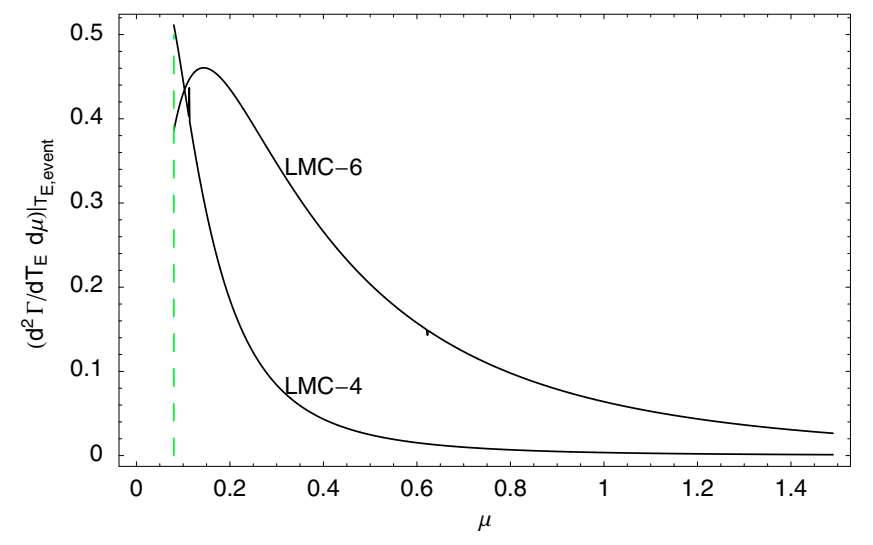

Fig. 11. Differential rate of the microlensing events with respect to the mass of the lens in the direction of events LMC -4 and LMC -6 . The dashed vertical line is at $\mu=0.08$. The $y$-axis values are in $10^{-3}$ units.

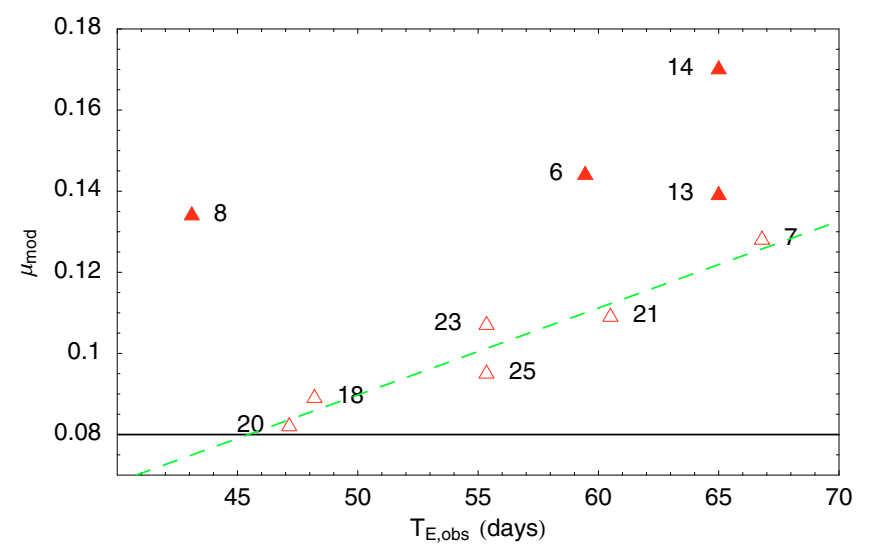

Fig. 12. Scatter plot of the modal value of $\mu$ (y-axis) with respect to the measured Einstein time ( $x$-axis) of each event. The label identifies the event. Filled (empty) triangles represent points with $\tau_{\mathrm{SL}}>$ $2 \times 10^{-8}\left(<2 \times 10^{-8}\right)$. The horizontal line indicates the lower limit of the lens mass for self-lensing, $\mu=0.08$. The four events LMC -1 , LMC -4, LMC-15 and LMC-27 have a modal value of the lens mass smaller than the lower limit and therefore are not shown. The dashed line represents the correlation line for the 6 events with $\tau_{\mathrm{SL}}<2 \times 10^{-8}$.

example we show in Fig. 11 this distribution calculated for the two events LMC-6 and LMC-4. LMC-6 is representative of the events for which the modal value $\mu_{\text {mod }}$ falls inside the selflensing mass interval 0.08-1.5. The second one has been chosen to demonstrate that there are also events for which $\mu_{\text {mod }}$ falls in the range $0-0.08$.

Figure 12 is the scatter plot between the measured $T_{\mathrm{E}}(x-$ axis) and the modal value of the lens mass $\mu=\frac{M_{1}}{M_{\odot}}$ ( $y$-axis), calculated for the distribution $\left(\frac{\mathrm{d}^{2} \Gamma}{\mathrm{d} T_{\mathrm{E}} \mathrm{d} \mu}\right)_{\varepsilon}$. In this case we prefer to use the modal value rather than the median, because it is independent of the choice of the amplitude of the interval of the allowed values of the lens masses, whereas the median value varies in accordance with this choice.

We find that the events LMC-1, LMC-4, LMC-15 and LMC-27 have a modal value of the lens mass smaller than the lower limit. We consider this result a strong indication that these events should be excluded from the self-lensing class. We have then calculated the linear correlation between $\mu_{\bmod }$ and $T_{\mathrm{E}}$ for the 6 remaining points of the cluster of ten. We find a high linear correlation, as shown by the dashed green straight line in Fig. 12 and by the calculated linear correlation coefficient, which is equal to 0.963 . We observe that the values of $\mu_{\text {mod }}$ of the six events range between 0.08 and 0.13 , an interval narrow enough to justify a linear approximation to represent a small portion of a parabolic curve, around which we expect the correlated points to disperse, bearing in mind that $T_{\mathrm{E}}$ is proportional to $\sqrt{\mu}$. The six linearly correlated events, therefore, constitute a homogeneous population, clearly distinct, also in this parameter space, from that formed by events with $\tau_{\mathrm{SL}}>2 \times 10^{-8}$ for which, for a given value of the observed Einstein time, we get significantly higher values for the mass.

We have also calculated the distributions $\left(\frac{\mathrm{d}^{2} \Gamma}{\mathrm{d} T_{\mathrm{E}} \mathrm{d} x}\right)_{\varepsilon}$ along the lines of sight pointing towards the 14 LMC microlensing events, in order to obtain a second independent check of the homogeneity of the cluster of events with $\tau_{\mathrm{SL}} \leq 2 \times 10^{-8}$. Figure 13 is the scatter plot between the measured $T_{E}$ ( $x$-axis) and the median (square boxes) of the parameter $\delta=\frac{D_{\mathrm{os}}-D_{\mathrm{ol}}}{D_{\mathrm{os}}}=$ $1-x$ ( $y$-axis), proportional to the distance between the lens and the source.

We find that the points of the first cluster have a high linear correlation, as shown by the calculated linear correlation coefficient, which is equal to 0.955 . We observe also that adding the event LMC-1 to the set of 9 and recalculating the linear correlation coefficient between $\delta$ and $T_{\mathrm{E}}$, we find a small increase, from 0.955 to 0.965 , suggesting that this event, lying inside the contour line at optical depth $\tau_{\mathrm{SL}}=2 \times 10^{-8}$, forms a homogeneous population with that of the 9 that lie outside this contour line. The ten events, represented by gray square boxes, are highly correlated as shown by the dashed green straight line in Fig. 13. This is, again, a strong indication that they constitute a homogeneous population of events. Together with the fact that, as shown in Fig. 10, the measured Einstein time fluctuates around a median value very different from the median Einstein times calculated with the self-lensing formulae, this allows us to exclude the possibility that this is a class of selflensing events. But, before any definitive assessment of the nature of these events can be made, we have to wait for an analogous statistical analysis of microlensing events due to lenses in the halos of the LMC or of our Galaxy: such an analysis is now underway.

We come now to the discussion of the changes in the microlensing rate induced by the different bar geometry configurations and velocity dispersions, as introduced in Sect. 3.2. The main point to be stressed is that the separation into the two populations for the events already identified is in these cases enhanced. Indeed, the expected characteristics change significantly only for those events along the lines of sight pointing towards the central region: LMC-6, LMC-8 and LMC-14, and, marginally, LMC-1 and LMC-27, as is the case for the selflensing optical depth. In Table 2 we report the predicted values for the Einstein time for these 5 events, the changes for the others being at most of $\sim 5 \%$ (in particular this is the case for the event LMC-13). As expected, an increase in the velocity dispersion for the bar component leads to a decrease ( 20\%) for the predicted values of the Einstein time. For the events 
Table 2. For each MACHO event located within or near the bar region, where the variation due to the change in the geometry turns out to be significant, we report for the Einstein time (days): the observed value and the predicted values for the three geometry configurations, bar coplanar with the disk, bar rotated by $40^{\circ}$ with respect to the disk plane and bar shifted by $0.5 \mathrm{kpc}$ along the line of sight towards the observer. The left values are for a velocity dispersion of the bar component of $\sigma_{\mathrm{b}}=20.2 \mathrm{~km} \mathrm{~s}^{-1}$ (as for the disk), whereas the right values are for $\sigma_{\mathrm{b}}=$ $30.0 \mathrm{~km} \mathrm{~s}^{-1}$. We report also the values for the self-lensing optical depth in the direction of the events.

\begin{tabular}{|c|c|c|c|c|c|c|c|}
\hline$\overline{\overline{\text { Event }}}$ & $\overline{\overline{T_{\mathrm{E}, \mathrm{obs}}}}$ & $\begin{array}{c}T_{\mathrm{E}, 50 \%} \\
\text { coplanar }\end{array}$ & $\begin{array}{l}T_{\mathrm{E}, 50 \%} \\
\text { rotated }\end{array}$ & $\begin{array}{c}T_{\mathrm{E}, 50 \%} \\
\text { shifted }\end{array}$ & $\begin{array}{l}\tau\left(10^{-8}\right) \\
\text { coplanar }\end{array}$ & $\begin{array}{l}\tau\left(10^{-8}\right) \\
\text { rotated }\end{array}$ & $\begin{array}{l}\overline{\tau\left(10^{-8}\right)} \\
\text { shifted }\end{array}$ \\
\hline 1 & 22.3 & $\begin{array}{ll}64 & 56\end{array}$ & $\begin{array}{ll}76 & 67\end{array}$ & $\begin{array}{ll}64 & 56\end{array}$ & 2.24 & 3.33 & 2.23 \\
\hline 6 & 59.5 & $55 \quad 47$ & $57 \quad 48$ & $62 \quad 53$ & 2.84 & 3.16 & 3.90 \\
\hline 8 & 43.1 & $48 \quad 37$ & $53 \quad 41$ & 5140 & 4.72 & 5.99 & 5.58 \\
\hline 14 & 65.0 & $51 \quad 41$ & $60 \quad 47$ & $56 \quad 44$ & 3.72 & 5.33 & 4.53 \\
\hline 27 & 32.8 & $\begin{array}{ll}67 & 59\end{array}$ & $75 \quad 67$ & 6961 & 1.75 & 2.29 & 1.94 \\
\hline
\end{tabular}

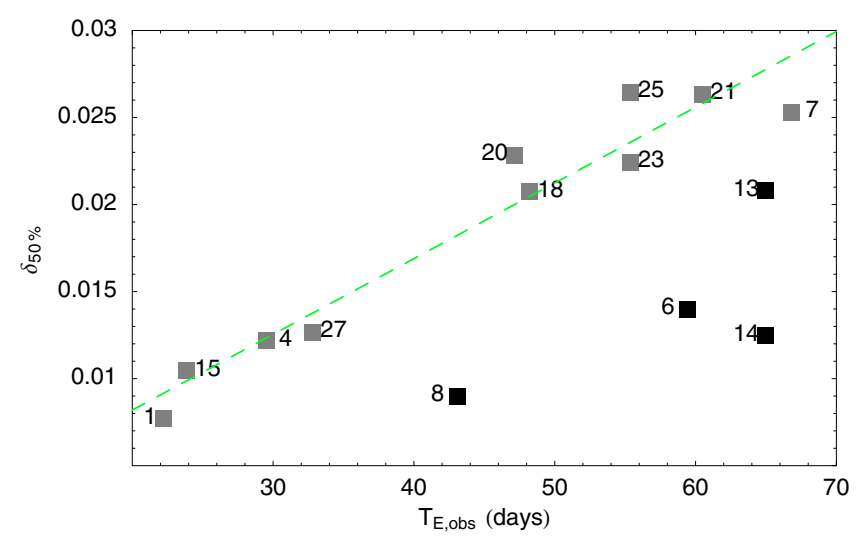

Fig. 13. Correlation plot of the median value (square boxes) of $\delta=$ $\frac{D_{\mathrm{os}}-D_{\mathrm{ol}}}{D_{\mathrm{os}}}(y$-axis) with respect to the measured Einstein time ( $x$-axis) of each event. The label identifies the event. The dashed line is the correlation straight line of the cluster of 10 events.

LMC-6, LMC-8 and LMC-14 the predicted values just fluctuate around the observed ones, while they remain substantially different for the events LMC-1 and LMC-27. The change in geometry leads, on the contrary, to a rise for the expected values of the Einstein time, of at most $\sim 10 \%$, for the events LMC6, LMC-8 and LMC-14; the effect is stronger for the rotated bar. For the event LMC-1 the difference between the observed and the predicted value is further enlarged (up to $\sim 16 \%$ ). This gives further support to the supposition that this short duration event is likely to belong to a different population. We note that the two opposite effects on the predicted Einstein time, the decrease linked to the rise of the velocity dispersion as opposed to the increase linked to the non-coplanar geometry, are actually to be expected, as $\Gamma \propto \tau / T$. Analogous considerations emerge from the analysis of the differential rate with respect to $x$ and the mass. In particular, for the mass, we note again two opposite effects. A rise induced by the increase in the velocity dispersion, and a decrease linked to the non-coplanar geometry. We observe a maximum increase of $\sim 15 \%$ for the event LMC-14.

To complete this analysis let us make a further point. In the differential rate, Eq. 17, we get an average result for all the possible configurations with lens and source either in the bar or in the disk of the LMC. This is of course in accordance with our approach aimed at the study of the statistical properties of the self-lensing population as a whole. However, it is in principle interesting to notice that the expected characteristics can be actually rather different depending on the configuration. As an example, we consider the event LMC-14, coplanar bar geometry, $\sigma_{\mathrm{b}}=20.2 \mathrm{~km} \mathrm{~s}^{-1}$. With respect to the previously obtained result, expected median value for the Einstein time $T_{\mathrm{E}, 50 \%}=51$ days, we get a lower value in the lens-source barbar configuration, $\sim 43$ days, a significantly higher value for the disk-disk configuration, $\sim 67$ days, and an intermediate one, $\sim 55$ days, for both mixed configurations, disk-bar and bar-disk. We recall that the observed value for $\mathrm{LMC}-14$ is $T_{\mathrm{E}}=65$ days.

\subsection{Expected number of self-lensing events}

We compute the "field exposure", $E_{\text {field }}$, defined, as in Alcock et al. (2000a), as the product of the number of distinct light curves per field and the relevant time span, paying attention to eliminating the field overlaps. Furthermore we calculate the distribution $\frac{\mathrm{d} \Gamma}{\mathrm{d} T_{\mathrm{E}}}$ along the line of sight pointing towards the center of each field. In this way we obtain the number of expected events for self-lensing, field by field, given by

$N_{\mathrm{SL}, \text { field }}=E_{\text {field }} \int_{0}^{\infty} \frac{\mathrm{d} \Gamma}{\mathrm{d} T_{\mathrm{E}}} \mathcal{E}\left(T_{\mathrm{E}}\right) \mathrm{d} T_{\mathrm{E}}$

where $\mathcal{E}\left(T_{\mathrm{E}}\right)$ is the detection efficiency.

In Fig. 14 we report in parentheses the expected number of events in each field we obtain by using the Chabrier exponential IMF. Summing over all fields we find that the expected total number of self-lensing events is $\sim 1.2$, while we would get $\sim 1.3$ with the the double power law IMF, in both cases roughly 1-2 events. Clearly, taking also into account the uncertainties in the parameter used following the van der Marel model for the LMC the actual number could also be somewhat higher but hardly more than twice our estimate. A conclusion we had already reached in Paper $\mathrm{I}$.

We have also computed the influence of the bar geometry and dispersion velocity on the number of expected events. We find at most an increase of $\sim 50 \%$, bar rotated and $\sigma_{\mathrm{b}}=$ $30 \mathrm{~km} \mathrm{~s}^{-1}$, which gives two to three events.

In conclusion, we have identified, according to their expected characteristics as compared to what is observed, a set of 4 possible events belonging to a self-lensing population (LMC6, LMC-8, LMC-13 and LMC-14). As already noted, there is some experimental evidence that the $\mathrm{LMC}-14$ is a self-lensing 
event (Alcock et al. 2001b). If this is true, our prediction of at most 2-3 such events seems to indicate that it is likely that some of the others are not due to self-lensing.

\section{Asymmetry}

In this section we consider from a different perspective the issue of the expected near/far asymmetry for events due to lenses located in the LMC or the MW halo. In particular we study, by means of a statistical analysis, the spatial distribution of the events, both in the framework of the van der Marel et al. (2002) picture and, for comparison, of the older one (e.g., Westerlund 1997). The aim here is to give further support to our main conclusion, namely that self-lensing, for which we do not expect any asymmetry in the spatial distribution, cannot explain all the detected events.

In Fig. 14, the 30 well-sampled fields analyzed by the MACHO collaboration (red squares), together with the 16 events $^{12}$, are plotted in a reference frame (black axes) centered on $\alpha_{\mathrm{CM}}=5^{\mathrm{h}} 27.6^{\mathrm{m}}, \delta_{\mathrm{CM}}=-69.87^{\circ}, \mathrm{J} 2000$. We also report the position (triangles) of the microlensing events found by the EROS collaboration (Lasserre et al. 2000) even if we do not consider them in the present analysis.

We divide the LMC field into three regions: a strip centered on the line of nodes and delimited by two parallel straight lines at a distance of $\approx 0.35 \mathrm{kpc}$ from the line of nodes, and two outer regions, belonging to the closer north-east side and to the farther south-west one. The amplitude of the central strip reflects the $1 \sigma$ uncertainties in the position of the center of mass of carbon stars, as calculated by van der Marel et al. (2002). The exclusion of a substantial part of the bar region implies that the following discussion is not affected by the different choices for the bar geometry.

The green dashed-dot line represents the line of nodes with a position angle $\Theta=129.9^{\circ}$ and the two green dashed lines delimit the two outer regions belonging respectively to the near and far part of the LMC.

In the same figure a second reference frame is also shown, representing the pre-van der Marel LMC models. The frame (light blue axes) is centered on $\alpha_{\text {gas }}=5^{\mathrm{h}} 17.6^{\mathrm{m}}, \delta_{\text {gas }}=-69^{\circ} 02^{\prime}$, J2000 (Kim et al. 1998), and the line of nodes has a position angle $\Theta_{\text {Gyuk }}=170^{\circ}$ (Gyuk et al. 2000). A region of exclusion, similar to the previous one will be considered also in this case, even if not drawn in the figure for clarity.

At first glance one observes that the distribution of the events shows a clear near-far asymmetry in the postvan der Marel geometry, namely, they are concentrated along the extension of the bar and in the south-west side of the LMC. On the contrary, the asymmetry is almost completely lost in the pre-van der Marel geometry, where the distribution reflects almost exactly the different weights of the observed fields in the two half planes, as we will show in the following.

The little empty circle in field number 5 shows the position of the baricenter of the $16 \mathrm{MACHO}$ events; its coordi-

12 The ensemble of 17 events discussed in Sect. 4 with the exclusion of LMC-22. nates in the post-van der Marel reference frame are $(0.96 \mathrm{kpc}$, $-0.02 \mathrm{kpc})$.

\subsection{Near-far asymmetry of the observed microlensing events in the $L M C$}

In order to give a quantitative analysis of the near-far asymmetry, we will concentrate on the MACHO observed fields (and the corresponding observed events) in the outer regions located on opposite sides of the line of the nodes and external to the dashed lines, which, at a good confidence level, belong respectively to the near and far part of the galaxy.

As a first point we determine the fraction of the MACHO fields included respectively in the north-east closer region "1" and in the south-west farther region "2", and we calculate in each the quantities $\Sigma_{1}$ and $\Sigma_{2}$, defined as the sum of the product of star number per field, and the corresponding observation time, where we count only once the stars in the overlapping part of the fields. The ratio $\Sigma_{i} /\left(\Sigma_{1}+\Sigma_{2}\right)$ gives us the probability $p_{i}$ that a microlensing event would fall in the first or second region, respectively. Note that the probability scheme so defined depends only on the global observation strategy in the near and far region (field distribution and observation time). We find $p_{1} \approx 0.31$ for the case of the post-van der Marel LMC geometry, and $\tilde{p}_{1} \approx 0.48$ for the pre-van der Marel LMC geometry.

We are interested in testing whether the observed events support the modelled probability schemes. The Pearson chisquare statistic $\mathcal{A}_{N}$ provides a non-parametric test for the comparison:

$\mathcal{A}_{N}=\sum_{i=1}^{2} \frac{\left(n_{i}-N p_{i}\right)^{2}}{N p_{i}}$,

where $n_{i}$ are the events falling in the $i$-region, and $N=n_{1}+n_{2}$. $\mathcal{A}_{N}$ is approximately distributed as chi-square with one degree of freedom. Small values of $\mathcal{A}_{N}$ tend to support the null hypothesis that the $p_{i}$ match the measured distribution.

In the post-van der Marel geometry we get, respectively, one and nine observed events in regions " 1 " and " 2 ", and then

$\mathcal{A}_{10}=2.11$.

On the contrary, in the case of pre-van der Marel geometry, we obtain:

$\tilde{\mathcal{A}}_{12}=0.22$,

starting from a set of five and seven observed events.

In the pre-van der Marel geometry $\mathcal{A}_{N}$ has a value near to zero. At the confidence level of $\simeq 64 \%$ the null hypothesis that the event distribution reflects almost exactly the weights of the two regions has to be accepted. This implies that the distribution of the lenses should be almost homogeneous along the lines of sight through the different regions of the LMC.

On the contrary, in the post-van der Marel geometry $\mathcal{A}_{N}$ assumes a value far enough from zero. At the confidence level of $\simeq 85 \%$ the null hypothesis must be rejected. This means that the observed asymmetry is greater than what is expected simply on the basis of the observational strategy. 


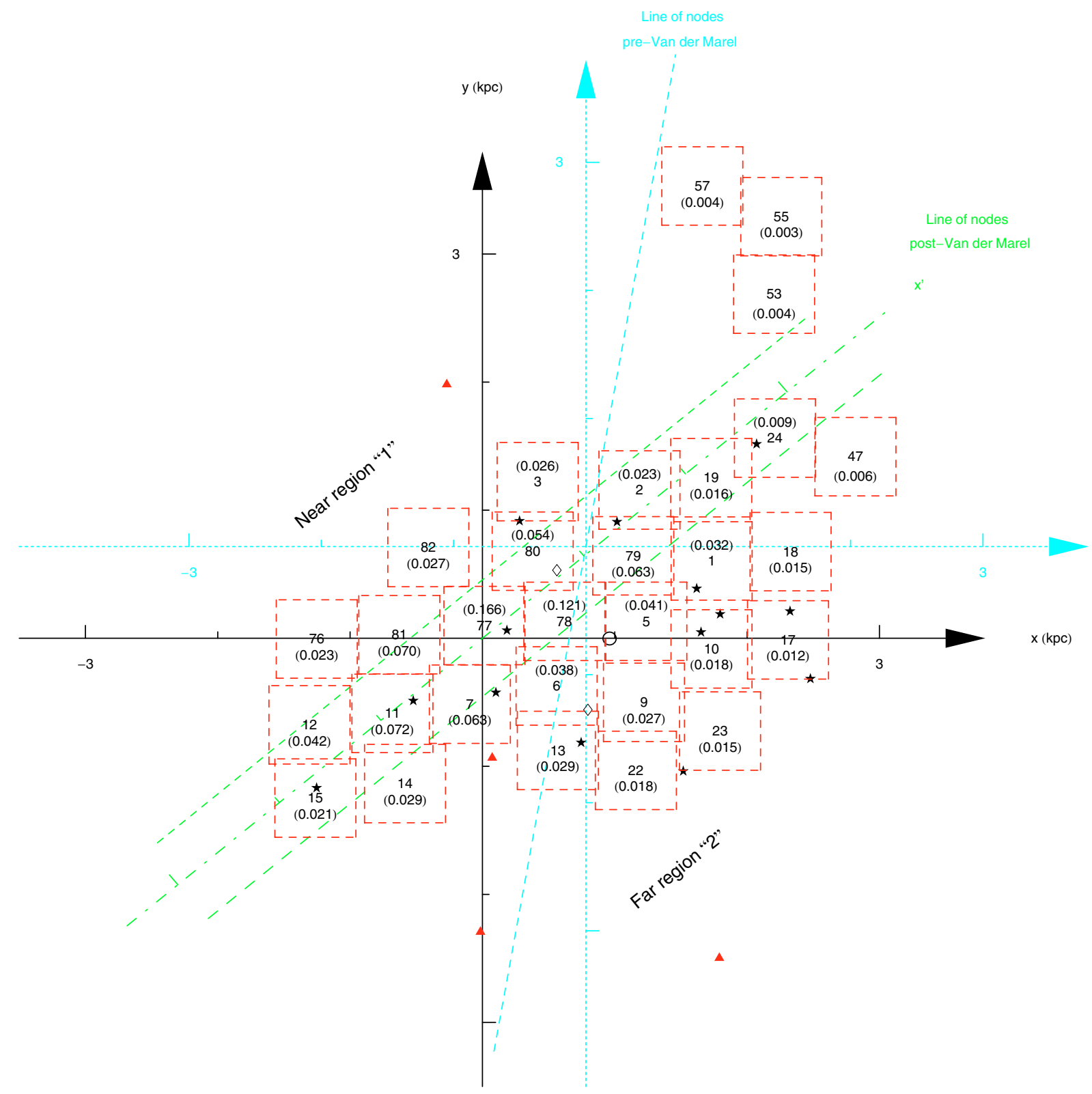

Fig. 14. Locations of the 30 MACHO fields with respect to post-Van der Marel LMC geometry (black reference axes) and with respect to preVan der Marel LMC geometry (light blue reference axes). Also shown are the locations of the MACHO and EROS microlensing candidates. The distances on the axes are in kpc. For each field, inside brackets, we report the expected number of self-lensing event as discussed in Sect. 4.4.

We are aware that these results have to be treated with caution inasmuch as the number of events is small. However, we note that the observed near-far asymmetry agrees with that induced by the inclination of the LMC disk already discussed in Sect. 4.1.

\section{An improved Gould inequality}

Gould's (1995) ingenious calculation provides two inequalities (Eqs. (2.8) and (3.3) of his paper), the second one involving the Jeans equation and the virial theorem, which allow, in some cases, a quick evaluation of an upper limit to the selflensing optical depth along a line of view through the center of a galaxy. In particular this was applied to the LMC disk, in order to exclude the Sahu (1994) hypothesis that the observed optical depth could be fully explained by self-lensing.

Our aim here is to obtain an improved version of the Gould inequality, and, at the same time, clarify its limits of applicability. We start from Eq. (10) and consider the case of a line of view passing through the center of the LMC.

Let us assume that the lens mass density $\rho_{1}$ is a function of a homogeneous polynomial of degree $n_{1}$ in the variables $(\xi, \eta, \zeta)$ of the reference frame defined in Sect. 3.1, and analogously that the star mass density $\rho_{\mathrm{s}}$ is a function of a homogeneous polynomial of degree $n_{\mathrm{s}}{ }^{13}$. Let us also denote by $z$ the current coordinate along the line of view through the center of the LMC disk, which we assume as the origin of the $z$-coordinate, and by

13 This is implicit in the Gould (1995) derivation. 
$i$ the disk inclination angle. Keeping in mind that for points belonging to a line of view passing through the center we have:

$\xi=0$

$\eta=z \sin i$

$\zeta=z \cos i$

we can write for the lens density distribution

$$
\begin{aligned}
\rho_{l}[0, \eta, \zeta] & =\rho_{l}\left[\sum_{k=0}^{n} c_{k, n-k}^{(1)}\left(\frac{\eta}{\eta_{1,0}}\right)^{k}\left(\frac{\zeta}{\zeta_{1,0}}\right)^{n-k}\right] \\
& =\rho_{1}\left[\gamma_{1}(i)\left(\frac{z}{\zeta_{1,0}}\right)^{n}\right]
\end{aligned}
$$

with

$\gamma_{1}(i)=\sum_{k=0}^{n} c_{k, n-k}^{(1)}\left(\frac{\eta_{1,0}}{\zeta_{1,0}}\right)^{k} \sin ^{k}(i) \cos ^{n-k}(i)$

and $\eta_{1,0}, \zeta_{1,0}$ the scale lengths of the density distribution of the lenses. Analogous expressions can be written for the star density distribution, changing the suffix "l" to "s".

From now on we follow Gould (1995), and put $D_{\mathrm{ol}}=d+z$ and $D_{\mathrm{os}}=d+w$, where $d$ is the distance, along the line of view, from the observer to the center of the disk. Taking into account that

$\frac{d+z}{d+w}<1$,

we obtain the following inequality for the optical depth:

$$
\begin{aligned}
\tau \leq & \frac{4 \pi G}{c^{2}} \int_{-t}^{t} \mathrm{~d} w \rho_{\mathrm{s}}\left[\gamma_{\mathrm{s}}(i)\left(\frac{w}{\zeta_{\mathrm{s}, 0}}\right)^{n_{\mathrm{s}}}\right] \\
& \times \frac{\int_{-d}^{w} \mathrm{~d} z(w-z) \rho_{\mathrm{l}}\left[\gamma_{1}(i)\left(\frac{z}{\zeta_{1,0}}\right)^{n_{1}}\right]}{\int_{-t}^{t} \mathrm{~d} w \rho_{\mathrm{s}}\left[\gamma_{\mathrm{s}}(i)\left(\frac{w}{\zeta_{\mathrm{s}, 0}}\right)^{n_{\mathrm{s}}}\right]}
\end{aligned}
$$

where $t$ is the LMC tidal radius.

We now integrate by parts twice, the first time obtaining:

$$
\begin{aligned}
\tau \leq & \frac{4 \pi G}{c^{2}} \int_{-t}^{t} \mathrm{~d} w \rho_{\mathrm{s}}\left[\gamma_{\mathrm{s}}(i)\left(\frac{w}{\zeta_{\mathrm{s}, 0}}\right)^{n_{\mathrm{s}}}\right] \\
& \times \frac{\int_{-d}^{w} \mathrm{~d} z \int_{-d}^{z} \mathrm{~d} u \rho_{l}\left[\gamma_{1}(i)\left(\frac{u}{\zeta_{1,0}}\right)^{n_{1}}\right]}{\int_{-t}^{t} \mathrm{~d} w \rho_{\mathrm{s}}\left[\gamma_{\mathrm{s}}(i)\left(\frac{w}{\zeta_{\mathrm{s}, 0}}\right)^{n_{\mathrm{s}}}\right]}
\end{aligned}
$$

and the second time obtaining:

$$
\begin{aligned}
\tau \leq & \frac{4 \pi G}{c^{2}} \int_{-d}^{t} \mathrm{~d} z \rho_{\mathrm{l}}\left[\gamma_{\mathrm{l}}(i)\left(\frac{z}{\zeta_{1,0}}\right)^{n_{1}}\right] \\
& \times\left\{\int_{-t}^{t} \mathrm{~d} w \frac{\int_{-d}^{w} \mathrm{~d} z \rho_{\mathrm{l}}\left[\gamma_{\mathrm{l}}(i)\left(\frac{z}{\zeta_{1,0}}\right)^{n_{1}}\right]}{\int_{-d}^{t} \mathrm{~d} z \rho_{\mathrm{l}}\left[\gamma_{\mathrm{l}}(i)\left(\frac{z}{\zeta_{1}}\right)^{n_{1}}\right]}\right. \\
& \left.\times\left(1-\frac{\int_{-t}^{w} \mathrm{~d} z \rho_{\mathrm{s}}\left[\gamma_{\mathrm{s}}(i)\left(\frac{z}{\zeta_{\mathrm{s}, 0}}\right)^{n_{\mathrm{s}}}\right]}{\int_{-t}^{t} \rho_{\mathrm{s}}\left[\gamma_{\mathrm{s}}(i)\left(\frac{z}{\zeta_{\mathrm{s}, 0}}\right)^{n_{\mathrm{s}}}\right] \mathrm{d} z}\right)\right\} .
\end{aligned}
$$

In case of self-lensing $\rho_{\mathrm{s}}$ and $\rho_{\mathrm{l}}$ coincide. Moreover, for distances higher than the tidal radius of the LMC, the star mass density $\rho_{\mathrm{s}}=0$, and we can move the lower integration limit from $-t$ or $-d$ to $-\infty$, and the upper from $t$ to $+\infty$. We are considering a line of view passing through the center of the LMC, therefore we can use the symmetry property of the mass density distributions with respect to the change of sign of the parameter $z$ and we obtain:

$$
\begin{aligned}
\tau \leq & \frac{\pi G}{c^{2}} \int_{-\infty}^{\infty} \mathrm{d} z \rho_{\mathrm{s}}\left[\gamma_{\mathrm{s}}(i)\left(\frac{z}{\zeta_{\mathrm{s}, 0}}\right)^{n_{\mathrm{s}}}\right] \\
& \times \int_{-\infty}^{\infty} \mathrm{d} v\left[1-F_{s}^{2}(v)\right],
\end{aligned}
$$

where

$F_{\mathrm{s}}(v)=\frac{2 \int_{0}^{v} \mathrm{~d} z \rho_{\mathrm{s}}\left[\gamma_{\mathrm{s}}(i)\left(\frac{z}{\zeta_{\mathrm{s}, 0}}\right)^{n_{\mathrm{s}}}\right]}{\int_{-\infty}^{\infty} \mathrm{d} z \rho_{\mathrm{s}}\left[\gamma_{\mathrm{s}}(i)\left(\frac{z}{\zeta_{\mathrm{s}, 0}}\right)^{n_{\mathrm{s}}}\right]}$

A suitable change of variables gives:

$$
\begin{aligned}
\tau \leq & \frac{2 \pi G \zeta_{\mathrm{s}, 0}^{2}}{c^{2}}\left[\gamma_{\mathrm{s}}(i)\right]^{-\frac{2}{n_{\mathrm{s}}}} \\
& \times \int_{-\infty}^{\infty} \rho_{\mathrm{s}}\left(u^{n_{\mathrm{s}}}\right) \mathrm{d} u \int_{0}^{\infty}\left[1-F_{\mathrm{s}}^{2}(v)\right] \mathrm{d} v,
\end{aligned}
$$

where

$F_{\mathrm{s}}(v)=\frac{2 \int_{0}^{v} \rho_{\mathrm{s}}\left(u^{n_{\mathrm{s}}}\right) \mathrm{d} u}{\int_{-\infty}^{\infty} \rho_{\mathrm{s}}\left(u^{n_{\mathrm{s}}}\right) \mathrm{d} u}$

and the integrations are now made on non-dimensional variables.

Let us observe that the inequality (29) can be applied for any inclination angle $i$ of the disk plane, not only for $i \ll \pi / 2$, as in Gould (1995), namely, we have no divergence problem for $i \rightarrow \pi / 2$. For instance, in the case of a double exponential disk, with scale lengths respectively equal to $R_{\mathrm{d}}$ and $\zeta_{\mathrm{d}}$, we obtain:

$$
\begin{aligned}
\tau \leq & \frac{2 \pi G \zeta_{\mathrm{d}}^{2}}{c^{2}} \frac{1}{\left[\cos (i)+\frac{\zeta_{\mathrm{d}}}{R_{\mathrm{d}}} \sin (i)\right]^{2}} \int_{-\infty}^{\infty} \rho_{\mathrm{s}}(u) \mathrm{d} u \\
& \times \int_{0}^{\infty}\left[1-F_{\mathrm{s}}^{2}(v)\right] \mathrm{d} v .
\end{aligned}
$$

Or in the case of a Gaussian profile of the mass density distribution for the LMC bar, as in Gyuk et al. (2000), with scale length $\eta_{\mathrm{b}}$ along the axis bar and $\zeta_{\mathrm{b}}$ in the orthogonal section, we obtain:

$$
\begin{aligned}
\tau \leq & \frac{2 \pi G \zeta_{\mathrm{b}}^{2}}{c^{2}} \frac{1}{\cos ^{2}(i)+\left(\frac{\zeta_{\mathrm{b}}}{\eta_{\mathrm{b}}}\right)^{2} \sin ^{2}(i)} \int_{-\infty}^{\infty} \rho_{\mathrm{s}}\left(u^{2}\right) \mathrm{d} u \\
& \times \int_{0}^{\infty}\left[1-F_{\mathrm{s}}^{2}(v)\right] \mathrm{d} v .
\end{aligned}
$$

For a bar having boxy contours and sharp edges as in the paper of Zhao \& Evans (2000), parameterized by an exponential profile with a fourth degree polynomial in the exponent, with scale length $\eta_{\mathrm{b}}$ along the axis bar and $\zeta_{\mathrm{b}}$ in the orthogonal section, we obtain:

$$
\begin{aligned}
\tau \leq & \frac{2 \pi G \zeta_{\mathrm{b}}^{2}}{c^{2}} \frac{1}{\sqrt{\cos ^{4}(i)+\left(\frac{\zeta_{\mathrm{b}}}{\eta_{\mathrm{b}}}\right)^{4} \sin ^{4}(i)}} \int_{-\infty}^{\infty} \rho_{\mathrm{s}}\left(u^{4}\right) \mathrm{d} u \\
& \times \int_{0}^{\infty}\left[1-F_{\mathrm{s}}^{2}(v)\right] \mathrm{d} v .
\end{aligned}
$$


Equation (29) is an improved version of Eq. (2.8) of Gould (1995).

Proceeding in the same way as in Gould (1995), we obtain the second Gould inequality, relating the optical depth to the mass-weighted velocity dispersion.

$\tau \leq 2 \frac{<v^{2}>}{c^{2}}\left[\gamma_{\mathrm{s}}(i)\right]^{-\frac{2}{n_{\mathrm{s}}}}$.

Let us note that these inequalities are based on two requirements:

- that the star density is a function of a homogeneous polynomial of the variables, of any degree;

- that the line of view passes through the center, so that we can use the reflection symmetry of the mass density distributions.

Let us note, moreover, that the inequalities (29) and (34) cannot be applied to the model of the LMC disk and bar we assumed in this paper, because the first property, that of homogeneity, is lacking.

It is therefore useful to derive a more general inequality, applicable to all kinds of density distribution.

\subsection{A more general inequality}

We start from Eq. (25), but we relax the requirement that the mass density distribution is a function of a homogeneous polynomial:

$\tau \leq \frac{4 \pi G}{c^{2}} \frac{\int_{-t}^{t} \mathrm{~d} w \rho_{\mathrm{s}}(w) \int_{-d}^{w} \mathrm{~d} z \int_{-d}^{z} \mathrm{~d} u \rho_{\mathrm{l}}(u)}{\int_{-t}^{t} \mathrm{~d} w \rho_{\mathrm{s}}(w)}$.

Let us divide in three parts the triangular region of integration in the plane $(u, z)$, delimited by the bisector of the first and third quadrant, by the parallel to the $u$-axis for which $z=w$ and by the parallel to the $z$-axis for which $u=-d$ : the first region is constituted by the triangle delimited by the bisector, the line $u=-d$ and the $u$-axis; the second by the rectangle delimited by the $u$-axis, the $z$-axis and the lines for which $z=w$ and $u=-d$; the third by the triangle delimited by the bisector, the $\mathrm{z}$-axis and the line for which $z=w$. Let us remember that $w$ can assume any value between $-t$ and $t$. In this way, the right hand side of Eq. (35) is given by the sum of three terms:

$$
\begin{array}{r}
\frac{4 \pi G}{c^{2}}\left\{\int_{-d}^{0} \mathrm{~d} z \int_{-d}^{z} \mathrm{~d} u \rho_{\mathrm{l}}(u)+\int_{-d}^{0} \mathrm{~d} z \rho_{\mathrm{l}}(z) \frac{\int_{-t}^{t} \mathrm{~d} w w \rho_{\mathrm{s}}(w)}{\int_{-t}^{t} \mathrm{~d} w \rho_{\mathrm{s}}(w)}\right. \\
\left.+\frac{\int_{-t}^{t} \mathrm{~d} w \rho_{\mathrm{s}}(w) \int_{0}^{w} \mathrm{~d} z \int_{0}^{z} \mathrm{~d} u \rho_{\mathrm{l}}(u)}{\int_{-t}^{t} \mathrm{~d} w \rho_{\mathrm{s}}(w)}\right\} .
\end{array}
$$

After an integration by parts, the first term becomes

$\frac{4 \pi G}{c^{2}} \int_{0}^{d} \mathrm{~d} z z \rho_{\mathrm{s}}(-z)$

the second term is null, thanks to the symmetry by reflection of $\rho_{\mathrm{s}}(z)$. As regards the third term we observe that for any $z$ belonging to the interval $(-t, t)$ we have

$\int_{0}^{z} \mathrm{~d} u \rho_{\mathrm{l}}(u) \leq \int_{0}^{t} \mathrm{~d} u \rho_{\mathrm{l}}(u)$ and therefore

$$
\begin{aligned}
\frac{\int_{-t}^{t} \mathrm{~d} w \rho_{\mathrm{s}}(w) \int_{0}^{w} \mathrm{~d} z \int_{0}^{z} \mathrm{~d} u \rho_{\mathrm{l}}(u)}{\int_{-t}^{t} \mathrm{~d} w \rho_{\mathrm{S}}(w)} \leq & \int_{-t}^{t} \mathrm{~d} w w \rho_{\mathrm{s}}(w) \\
& \times \frac{\int_{0}^{t} \mathrm{~d} u \rho_{\mathrm{l}}(u)}{\int_{-t}^{t} \mathrm{~d} w \rho_{\mathrm{s}}(w)}
\end{aligned}
$$

In this way we obtain that the third term is also less than zero. In conclusion we obtain the searched for inequality for the optical depth, valid for any mass density distribution of lenses. In particular for lenses in the Galactic halo we obtain:

$\tau_{\mathrm{GH}} \leq \frac{4 \pi G}{c^{2}} \int_{0}^{d} \mathrm{~d} z z \rho_{\mathrm{l}, \mathrm{GH}}(-z)$,

for lenses in the LMC halo we obtain

$\tau_{\mathrm{LMCH}} \leq \frac{4 \pi G}{c^{2}} \int_{0}^{t} \mathrm{~d} z z \rho_{\mathrm{l}, \mathrm{LMCH}}(z)$,

and for self-lensing we obtain

$\tau_{\mathrm{SL}} \leq \frac{4 \pi G}{c^{2}} \int_{0}^{t} \mathrm{~d} z z \rho_{\mathrm{s}}(z)$.

Applying this last inequality to the LMC mass density distribution used in our (coplanar) model we find

$\tau_{\mathrm{SL}} \leq 6 \times 10^{-8}$

only about $20 \%$ higher than the calculated value.

Notice that on the right hand side of the inequality (38) $\rho_{\mathrm{l}, \mathrm{GH}}(-z) \neq \rho_{\mathrm{l}, \mathrm{GH}}(z)$, since the Galactic halo density is not symmetric with respect to the LMC center. The inequality, therefore, cannot be obtained by a trivial dropping of the factor $(1-z)$ in the integrand of the expression defining the Galactic halo optical depth.

\section{Summary}

The great interest in the location of the observed microlensing events towards the LMC is motivated by the need to give an answer to the question of their nature. Namely, whether all the events can be attributed to known (luminous) populations, which would then exclude the possibility for a dark component in the halo in the form of MACHOs.

In this paper we are mainly concerned with the possible self-lensing origin of the observed microlensing events. In particular we have considered the results of the MACHO survey. We use the recent picture of the LMC disk given by van der Marel et al. (2002), and we explore different geometries for the bar component, as well as a reasonable range for the velocity dispersion of the bar population.

One interesting feature, essentially linked to the assumed disk geometry, is an evident near-far asymmetry of the optical depth for lenses located in the LMC Halo (this is not expected, with the possible exception of the inner region, for the self-lensing population). Indeed, similarly to the case of M31 (Crotts 1992; Jetzer 1994), and as first pointed out by Gould (1993), since the LMC disk is inclined, the optical depth is 
higher along lines of sight passing through larger portions of the LMC halo. We show that such a spatial asymmetry, beyond the one expected from the observational strategy alone, is indeed present in the observed events. With the caution suggested by the small number of detected events on which this analysis is based, this can be looked at, as observed by Gould (1993), as a signature of the presence of an extended halo around the LMC.

In the central region the microlensing signatures are strongly dependent on the assumed bar geometry. In particular, we have studied the variation (which can be as large as $50 \%$ ) in the self-lensing optical depth due to different geometries of the bar. However, the available data do not allow us to meaningfully explore this aspect in more detail.

As a further step in our analysis, we have studied the microlensing rate. Keeping in mind the observation of Evans \& Kerins (2000) that the timescale distribution of the events and their spatial variation across the LMC disk offers possibilities of identifying the dominant lens population, we have carefully characterized the ensemble of observed events on the hypothesis that all of them do belong to the self-lensing population. Through this analysis we have been able to identify a large subset of events that cannot be accounted as part of this population. The introduction of a bar component that is non-coplanar with respect to the disk turns out to strengthen this result. Again, the small number of events at our disposal does not yet allow us to draw definite conclusions, although the various arguments mentioned above are all consistent and converge quite clearly in the direction of excluding self-lensing as being the major source of the events.

Once more observations will be available, as will hopefully be the case with the SuperMacho experiment under way (Stubbs et al. 2002), the use of the above outlined methods can give a definitive answer to the problem of the location of the MACHOs and thus also to their nature.

Acknowledgements. The authors wish to thank the anonymous referee for his comments which improved the quality of this work and Chiara Mastropietro for useful discussion on the LMC morphology. L.M. and S.C.N. are partially supported by the Swiss National Science Foundation and S.C.N. is also partially supported by the Tomalla Foundation. G.S. wishes to thank the Institute of Theoretical Physics of the University of Zürich for the kind hospitality.

\section{References}

Alcock, C., Allsman, R. A., Alves, D. R., et al. 1997, ApJ, 486, 697 Alcock, C., Allsman, R. A., Alves, D. R., et al. 2000a, ApJ, 542, 281
Alcock, C., Allsman, R. A., Alves, D. R., et al. 2000b, ApJ, 541, 270

Alcock, C., Allsman, R. A., Alves, D. R., et al. 2001a, Nature, 414, 617

Alcock, C., Allsman, R. A., Alves, D. R., et al. 2001b, ApJ, 552, 259

Alcock, C., Allsman, R. A., Alves, D. R., et al. 2001c, ApJ, 552, 582

Alves, D. R., \& Nelson C. A., 2000, ApJ, 542, 789

Aubourg, E., Palanque-Delabrouille N., Salati, P., et al. 1999, A\&A, 347,850

Chabrier, G. 2001, ApJ, 554, 1274

Cioni, M.-R. L., Loup, C., Habing, H. J., et al. 2000, A\&AS, 144, 235

Crotts, A. P. S. 1992, ApJ, 399, L43

Cutri, R. M., Skrutskie, M. F., Van Dyk, S., et al. 2000, 2MASS Second Incremental Data Release

Drake, A. J., Cook, K. H., \& Keller, S. C. [arXiv: astro-ph/0404285]

de Rújula, A., Jetzer, Ph., \& Massó, E. 1991, MNRAS, 250, 348

de Rújula, A., Giudice, G. F., \& Mollerach, S., \& Roulet, E. 1995, MNRAS, 275, 545

Evans, N. W., \& Kerins, E. 2000, ApJ, 529, 917

Gerhard, O. E. 1993, MNRAS, 265, 213

Gould, A. 1993, ApJ, 404, 451

Gould, A. 1995, ApJ, 441, 77

Gould, A. 2004, ApJ, 606, 319

Griest K. 1991, ApJ, 366, 412

Gyuk, G., Dalal, N. \& Griest, K. 2000, ApJ, 535, 90

Jetzer, Ph. 1994, A\&A, 286, 426

Jetzer, Ph., Mancini, L. \& Scarpetta, G. 2002, A\&A, 393, 129 (Paper I)

Kim, S., Staveley-Smith, L., Dopita, M. A., et al. 1998, ApJ, 503, 674

Lasserre, T., Afonso, C., Albert J. N., et al. 2000, A\&A, 355, L39

Milsztajn, A. 2003, private communication

Nikolaev, S., Drake, A. J., Keller, S. C., et al. 2004, ApJ, 601, 260

Olsen, K. A. G., \& Salyk, C. 2002, AJ, 124, 2045

Primack, J. R., Seckel, D., \& Sadoulet, B. 1988, Ann. Rev. Nucl. Part. Sci., 38,751

Sahu, K. C. 1994, PASP, 106, 942

Stubbs, C. W., Rest, A., Miceli, A., et al. 2002, BAAS, 201, \#78.07

van der Marel, R. P., \& Franx, M. 1993, ApJ, 407, 525

van der Marel, R. P., \& Cioni, M. R. 2001, AJ, 122, 1807

van der Marel, R. P. 2001, AJ, 122, 1827

van der Marel, R. P., Alves, D. R., Hardy, E., \& Suntzeff, N. B. 2002, AJ, 124, 2639

von Hippel, T., Sarajedini, A., Ruiz, M. T. 2003, ApJ, 595, 794

Weinberg, M. D. 2000, ApJ, 532, 922

Weinberg, M. D., \& Nikolaev, S. 2001, ApJ, 548, 712

Westerlund, B. E. 1997, The Magellanic Clouds (Cambridge: Cambridge Univ. Press)

Wu, X. P. 1994, ApJ, 435, 66

Zasov, A. V., \& Khoperskov, A. V. 2002, Astron. Rep., 4603, 173

Zhao, H. S., \& Evans, N. W. 2000, ApJ, 545, L35 